\title{
Revisiting the dust properties in the molecular clouds of the Large Magellanic Cloud
}

\author{
D. Paradis ${ }^{1,2}$, C. Mény ${ }^{1,2}$, M. Juvela ${ }^{3}$, A. Noriega-Crespo ${ }^{4}$, and I. Ristorcelli ${ }^{1,2}$ \\ ${ }^{1}$ CNRS, IRAP, 9 Av. du Colonel Roche, BP 44346, 31028 Toulouse, cedex 4, France \\ e-mail: deborah.paradis@irap.omp.eu \\ ${ }^{2}$ UPS-OMP, IRAP, Université de Toulouse, Toulouse, France \\ ${ }^{3}$ Department of Physics, PO Box 64, 00014, University of Helsinki, Finland \\ ${ }^{4}$ Space Telescope Science Institute, 3700 San Martin Drive, Baltimore, MD 21218, USA
}

Received 29 January 2019 / Accepted 15 May 2019

\begin{abstract}
Context. Some Galactic molecular clouds show signs of dust evolution as compared to the diffuse interstellar medium, most of the time through indirect evidence such as color ratios, increased dust emissivity, or scattering (coreshine). These signs are not a feature of all Galactic clouds. Moreover, molecular clouds in the Large Magellanic Cloud (LMC) have been analyzed in a previous study based on Spitzer and IRIS data, at 4' angular resolution, with the use of one single dust model, and did not show any signs of dust evolution. Aims. In this present analysis we investigate the dust properties associated with the different gas phases (including the ionized phase this time) of the LMC molecular clouds at $1^{\prime}$ angular resolution (four times greater than the previous analysis) and with a larger spectral coverage range thanks to Herschel data. We also ensure the robustness of our results in the framework of various dust models.

Methods. We performed a decomposition of the dust emission in the infrared (from 3.6 to $500 \mu \mathrm{m}$ ) associated with the atomic, molecular, and ionized gas phases in the molecular clouds of the LMC. The resulting spectral energy distributions were fitted with four distinct dust models. We then analyzed the model parameters such as the intensity of the radiation field and the relative dust abundances, as well as the slope of the emission spectra at long wavelengths.

Results. This work allows dust models to be compared with infrared data in various environments for the first time, which reveals important differences between the models at short wavelengths in terms of data fitting (mainly in the polycyclic aromatic hydrocarbon bands). In addition, this analysis points out distinct results according to the gas phases, such as dust composition directly affecting the dust temperature and the dust emissivity in the submillimeter and different dust emission in the near-infrared (NIR).

Conclusions. We observe direct evidence of dust property evolution from the diffuse to the dense medium in a large sample of molecular clouds in the LMC. In addition, the differences in the dust component abundances between the gas phases could indicate different origins of grain formation. We also point out the presence of a NIR-continuum in all gas phases, with an enhancement in the ionized gas. We favor the hypothesis of an additional dust component as the carrier of this continuum.
\end{abstract}

Key words. dust, extinction - infrared: ISM - submillimeter: ISM - Magellanic Clouds

\section{Introduction}

The study of molecular clouds is important to understand the process of star formation. Whereas some clouds show activity of high-mass star formation, others seem to be quiescent and preferentially lead to low-mass star formation or to no star formation at all (Lis et al. 2001). It is therefore assumed that young stars and HII regions are born inside the molecular clouds. Ionized gas is then often located in the vicinity of the clouds. We therefore expect to observe changes in the properties of dust associated with the molecular clouds, and especially in the different phases of the gas inside and surrounding the cloud. Indeed, the dense molecular phase could be shielded from the interstellar radiation field and ensure the survival of small grains and/or molecules in this phase. In addition, ice mantles could cover the largest grain surfaces and allow dust aggregation processes. However, aggregation is not directly observable but could be linked to the decrease of the IRAS $60 / 100 \mu \mathrm{m}$ observed in some clouds (Laureijs et al. 1991), potentially resulting from the disappearance of the small grains. Changes in the dust emissivity have been observed in some molecular clouds (Stepnik et al. 2003; Paradis et al. 2009) and could be the consequence of grain coagulations. Recently, Ysard et al. (2018) analyzed the effect of grain growth by comparing the dust properties derived for isolated grains and aggregates, using Mie theory and Discrete Dipole Approximation (DDA) calculations. They evidenced a far-infrared (FIR) dust-mass opacity increase due to the porosity induced by coagulation. Moreover, in the recent Planck Catalogue of Galactic Cold Clumps (Planck Collaboration XXVIII 2016), the authors determined a mean spectral index of 1.9 when considering all clumps with a good quality of flux, and 2.1 for clumps with temperatures below $14 \mathrm{~K}$. These values are larger than the mean value of $\sim 1.6$ obtained for the diffuse Galactic emission (Planck Collaboration XI 2014).

Paradis et al. (2011a) performed a first statistical analysis of the 272 molecular clouds of the Large Magellanic Cloud (LMC) by decomposing the dust emission observed with Spitzer and IRAS into the atomic and molecular gas phases (using ATCA/Parkes HI and NANTEN ${ }^{12} \mathrm{CO}$ data). This study did not allow the identification of any statistical changes in the dust properties between the gas phases, as opposed to some studies of Galactic molecular clouds (Stepnik et al. 2003; Paradis et al. 2009; Ysard et al. 2013). However, this analysis was limited in longer wavelengths to $160 \mu \mathrm{m}$ and was done at $4^{\prime}$ angular 
resolution, corresponding to spatial scales of $60 \mathrm{pc}$ at the distance of the Large Magellanic Cloud $(\simeq 50 \mathrm{kpc}$, Keller \& Wood 2006; Feast 1999).

In this present paper, we perform a similar analysis as in Paradis et al. (2011a) but at $1^{\prime}$ angular resolution (corresponding to spatial scales of $\simeq 15 \mathrm{pc}$ ), thanks to the Mopra ${ }^{12} \mathrm{CO}$ data obtained as part of the MAGMA project (Wong et al. 2011). At this angular resolution we expect to investigate dust properties in cold-enough environments. Spitzer data combined to Herschel data allow the spectral range to be extended to the submillimeter (submm) domain, crucial to observe any potential changes in the slope of the dust emission spectra. Moreover, the ionized gas phase is included in the dust emission decomposition. The spectral energy distributions (SEDs) are modeled using different dust models: Jones et al. (2013, hereafter AJ13), Compiègne et al. (2011, hereafter MC11), Draine \& Li (2007, hereafter DL07) and an improved version of the Désert et al. (1990, hereafter DBP90) model. This was done to avoid results that would be strongly model dependent. This work also allows the different dust models developed to reproduce the diffuse interstellar medium (ISM) to be tested in several environments characterized by different densities and temperatures. After a brief description of the data sets in Sect. 2, we explain in Sect. 3 the methodology we adopt to decompose the dust emission in the different gas phases. Sections 4 and 5 present the dust models we use here, as well as their fitting residuals compared to the observations. In Sect. 6 we analyze the submm behavior of the SEDs related to the gas phases. We then describe and discuss the results of this work in Sects. 7 and 8. Conclusions are given in Sect. 9.

\section{Observations}

\subsection{Infrared data}

\subsubsection{Spitzer}

In order to trace the IR emission from 3.6 to $160 \mu \mathrm{m}$, we use data from two instruments on board the Spitzer satellite: the Infrared Array Camera (IRAC; Fazio et al. 2004) and the Multiband Imaging Photometer for Spitzer (MIPS; Rieke et al. 2004). These data were obtained as part of the SAGE (Surveying the Agents of Galaxy Evolution) Spitzer legacy survey (Meixner et al. 2006), covering the entire LMC. The IRAC observed at $3.6,4.5,5.8$, and $8 \mu \mathrm{m}$ with an angular resolution ranging from 1.6 to $1.9^{\prime \prime}$. The MIPS provided images at 24,70 , and $160 \mu \mathrm{m}$ at an angular resolution of 6,18 , and $40^{\prime \prime}$, respectively. Only the 24 and $160 \mu \mathrm{m}$ MIPS data have been used whereas MIPS data at $70 \mu \mathrm{m}$ have been replaced by the PACS $70 \mu \mathrm{m}$ data (see following section).

We apply the photometric correction to the IRAC maps by multiplying them by $0.737,0.772,0.937$, and 0.944 (from 3.6 to $8 \mu \mathrm{m})$ to account for the difference between calibration on pointsource and extended sources (Reach et al. 2005). We assume a $10 \%$ calibration uncertainty for all Spitzer data, taking the repeatability of measurements into account in the uncertainty. We then make the quadratic sum of the absolute calibration error and the statistical errors computed as the standard deviation in a region with low emission, that is, the entire region located outside a ring of $4^{\circ}$ in radius from the center of the maps $\left(\alpha_{2000}=05^{\mathrm{h}} 18^{\mathrm{m}} 48.0^{\mathrm{s}}, \delta_{2000}=-68.7^{\circ}\right)$.

\subsubsection{Herschel}

Dust emission in the FIR to the submm domain was mapped using the Herschel/PACS (70 and $100 \mu \mathrm{m})$ and SPIRE (250,

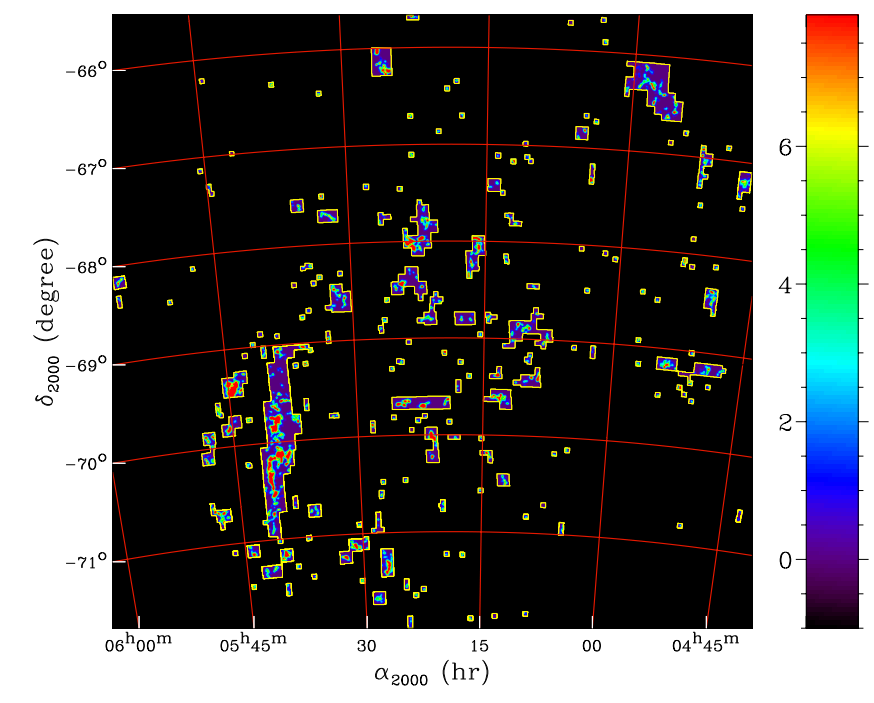

Fig. 1. Mopra ${ }^{12} \mathrm{CO}$ integrated intensity survey (in $\mathrm{K} \mathrm{km} \mathrm{s}^{-1}$ ) of molecular clouds in the LMC, as part of the MAGMA project (Wong et al. 2011).

350 and $500 \mu \mathrm{m})$ instruments, as part of the Heritage program (Meixner et al. 2010). The angular resolution of the original data is between 5 and $36^{\prime \prime}$. We take absolute uncertainties of $10 \%$ for PACS (Poglitsch et al. 2010) and 7\% for SPIRE (observer manual v2.4). The white noise computed in the same way as for Spitzer data is added in quadrature.

The OI $63 \mu \mathrm{m}$ (or OIII 58 and $88 \mu \mathrm{m}$ ) line could contaminate the 70 and $100 \mu \mathrm{m}$ bands. Its contribution to the MIPS $70 \mu \mathrm{m}$ band has been studied in the LMC by Bernard et al. (2008). The line should be very bright to significantly contribute to the brightness at $70 \mu \mathrm{m}$. Some [CII] $158 \mu \mathrm{m}$ and [OI] $145 \mu \mathrm{m}$ line emissions should also have been visible in the MIPS $160 \mu \mathrm{m}$, which were not observed. We therefore do not expect significant contributions from $\mathrm{O}$ or $\mathrm{C}$ lines.

\subsection{Gas tracers}

\subsubsection{HI emission}

As a tracer of the atomic gas, we use the Kim et al. (2003) $21 \mathrm{~cm}$ map, at a spatial resolution of $1^{\prime}$, which is a combination of interferometric data from the Australia Telescope Compact Array (ATCA; $1^{\prime}$ ), and the Parkes antenna (15.3'; Staveley-Smith et al. 2003). The HI data were integrated in the velocity range $190 \mathrm{~km} \mathrm{~s}^{-1}<V_{\mathrm{LSR}}<386 \mathrm{~km} \mathrm{~s}^{-1}$. The HI integrated intensity map has been converted to HI column density $\left(N_{\mathrm{H}}^{\mathrm{HI}}\right)$ by applying the standard conversion factor, $X_{\mathrm{HI}}$, equal to $1.82 \times$ $10^{18} \mathrm{H} \mathrm{cm}^{-2}\left(\mathrm{~K} \mathrm{~km} \mathrm{~s}^{-1}\right)^{-1}$, (Spitzer 1978; Lee et al. 2015).

\subsubsection{CO emission}

As a tracer of the molecular gas, we use the latest release of the data obtained with the $22 \mathrm{~m}$ Mopra telescope of the Australia Telescope National Facility, as part of the MAGMA project (Wong et al. 2011). The MAGMA LMC survey is a follow-up to the NANTEN survey at a resolution of $\simeq 1^{\prime}$ (see Fig. 1). The integrated intensity maps $\left(W_{\mathrm{CO}}\right)$ are converted to molecular column densities using the relation:

$N_{\mathrm{H}_{2}}=X_{\mathrm{CO}} W_{\mathrm{CO}}$,

with $X_{\mathrm{CO}}$ being the CO-to- $\mathrm{H}_{2}$ conversion factor. Hughes et al. (2010) found an average value of $4.7 \times 10^{20} \mathrm{H} \mathrm{cm}^{-2}\left(\mathrm{~K} \mathrm{Km} \mathrm{s}^{-1}\right)^{-1}$ 
for the LMC MAGMA clouds. Leroy et al. (2011) determined a value of $3 \times 10^{20} \mathrm{H} \mathrm{cm}^{-2}\left(\mathrm{~K} \mathrm{~km} \mathrm{~s}^{-1}\right)^{-1}$ in the LMC, whereas Roman-Duval et al. (2014) found upper limits to be $6 \times 10^{20} \mathrm{H} \mathrm{cm}^{-2}\left(\mathrm{~K} \mathrm{~km} \mathrm{~s}^{-1}\right)^{-1}$. Taking the dispersion of values into account, we decided to adopt an intermediate value equal to $4 \times 10^{20} \mathrm{H} \mathrm{cm}^{-2}\left(\mathrm{~K} \mathrm{~km} \mathrm{~s}^{-1}\right)^{-1}$.

\subsection{3. $\mathrm{H} \alpha$ emission}

The warm ionized gas, with an electron temperature $T_{\mathrm{e}} \simeq 10^{4} \mathrm{~K}$, emits both recombination lines and radio free-free continuum. The $\mathrm{H} \alpha$ line is the brightest of the recombination lines. To trace the ionized gas we use the Southern H-Alpha Sky Survey Atlas (SHASSA, Gaustad et al. 2001) data set which covers the southern hemisphere $\left(\delta<15^{\circ}\right)$. The angular resolution of the data is $0.8^{\prime}$, and the sensitivity is 2 Rayleigh per pixel $(1$ Rayleigh $=$ $10^{6}$ photons $\mathrm{cm}^{-2} \mathrm{~s}^{-1}$ ). By assuming a constant electron density $n_{\mathrm{e}}$ along each line of sight, we derive the $\mathrm{H}^{+}$column density with the relation (Lagache et al. 1999):

$\frac{N_{\mathrm{H}}^{\mathrm{H}^{+}}}{\mathrm{H} \mathrm{cm}^{-2}}=1.37 \times 10^{18} \frac{I_{\mathrm{H} \alpha}}{\mathrm{R}} \frac{n_{\mathrm{e}}}{\mathrm{cm}^{-3}}$,

where 1 Rayleigh $=2.25 \mathrm{pc} \mathrm{cm}^{-6}$ for $T_{\mathrm{e}}=8000 \mathrm{~K}$ (see, for instance Dickinson et al. 2003).

To determine the electron density we first compute the mean emission measure $\left(\mathrm{EM} ; 1 \mathrm{EM}=2.75 T_{4}^{0.9} I(\mathrm{H} \alpha) \mathrm{cm}^{-6} \mathrm{pc}\right.$ ) for all pixels associated with molecular clouds, by assuming an electron temperature of $8000 \mathrm{~K}$. As in Paradis et al. (2011b) we adopt a vertical extent of $\mathrm{H}^{+}$of $500 \mathrm{pc}$. We then obtain an electron density $n_{\mathrm{e}}$ close to $1 \mathrm{~cm}^{-3}$. This value is likely an upper limit since the vertical extent of $\mathrm{H}^{+}$is approximate and could be as high as the estimated scale height in our Galaxy ranging between 1000 and 1800 pc. By comparison, Paradis et al. (2011b) derived a value of $n_{\mathrm{e}}=0.055 \mathrm{~cm}^{-3}$ for the diffuse ionized gas of the LMC, $n_{\mathrm{e}}=1.52 \mathrm{~cm}^{-3}$ for typical HII regions and $n_{\mathrm{e}}=3.98 \mathrm{~cm}^{-3}$ for bright HII regions.

\subsection{Additional data processing}

\subsubsection{Galactic foreground}

Infrared data of the LMC can be significantly affected by Galactic foreground emission. The IR foreground contribution at all Spitzer and Herschel wavelengths was subtracted from the maps using the HI foreground map constructed by Staveley-Smith et al. (2003) at an angular resolution of $14^{\prime}$. Parkes HI data have been integrated in the velocity range $-64<v_{\mathrm{LSR}}<100 \mathrm{Km} \mathrm{s}^{-1}$, excluding emission coming from the LMC itself. The IR foreground contribution from 3.6 to $100 \mu \mathrm{m}$ has been taken from Bernard et al. (2008), using the IR brightness values corresponding to the best fit of the average high-latitude Galactic emission SED of Dwek et al. (1997). The contribution in the Herschel SPIRE bands has been derived from a modified black body.

\subsubsection{Star removal}

IRAC maps are highly affected by stellar emission. However, to perform a rigorous analysis, we removed the stellar contribution in all Spitzer and Herschel maps. We followed the exact same procedure as described in Bernard et al. (2008).

\subsubsection{Convolution to 1 arcmin}

To compare different data sets, all maps need to be convolved to a common resolution, set to the lowest resolution of the data sets, which is $1^{\prime}$ here. We used a Gaussian kernel with $\theta_{\text {FWHM }}=$ $\sqrt{\left(\left(1^{\prime}\right)^{2}-\left(\theta_{\mathrm{FWHM}}^{\mathrm{d}}\right)^{2}\right)}$, with $\theta_{\mathrm{FWHM}}^{\mathrm{d}}$ the original resolution of the
maps.

\section{Decomposition of the dust emission}

We perform correlations between IR emission and gas tracers for each molecular cloud. We use the same cloud classification as described in the Fukui et al. (2008) catalog, which accounts for 272 molecular clouds. Each cloud observed with Mopra is extended by 4 pixels (with a pixel size of $14^{\prime \prime}$ ) to include HI and $\mathrm{H} \alpha$ gas surrounding the clouds. We then decompose the IR-submm emission at each wavelength (from 3.6 to $500 \mu \mathrm{m}$ ), such as:

$I_{v}(\lambda)=a_{v}(\lambda) N_{\mathrm{H}}^{\mathrm{HI}}+b_{v}(\lambda) N_{\mathrm{H}}^{\mathrm{CO}}+c_{v}(\lambda) N_{\mathrm{H}}^{\mathrm{H}^{+}}+d_{v}(\lambda)$,

where the $a, b, c$, parameters denote the emissivity associated with each phase of the gas, and $d$ is a constant that could represent an additional gas phase such as the dark gas component, and/or possible offsets in the data. We obtain a SED representative of each phase of the gas, for each molecular cloud.

In Paradis et al. (2011a) the dark gas component had been included in the decomposition. We reconstructed a dark gas map at a resolution of the $1^{\prime}$ following the method of Bernard et al. (2008) and adopting the $X_{\mathrm{CO}}$ value defined in our analysis, and performed the decomposition. This new decomposition significantly reduced the quality of the obtained SEDs, that is, it gives more negative correlations. It significantly reduces the statistics of this analysis by excluding many clouds. Moreover, the dark gas component is highly correlated to the atomic gas since this component is used when constructing the dark gas map. For all these reasons, we decided to present results obtained when we do not include the dark gas. The main impact of the absence of dark gas in the decomposition is on the absolute levels of gas column densities (especially in the atomic phase), and as a consequence in the absolute levels of the dust abundances. The effect does not have significant consequences for the dust relative abundances. We carefully checked the consistency of all our results with and without the dark phase and can ensure that the conclusions are identical (see Sect. 7.2 and the appendix for a comparison of the results).

\section{Modeling}

\subsection{Description of the models}

The DustEM package ${ }^{1}$ offers the possibility to use four different dust models:

- A version of the Désert et al. (1990) model, that consists in three dust components: the polycyclic aromatic hydrocarbons (PAHs), the very small grains (VSGs) probably carbon dominated, and the big grains (BGs) probably made of a size distribution of silicates with some dark refractory mantles. The original model was updated to reproduce the actual shape of the PAH emission features as derived from ISO and Spitzer spectroscopy measurements and to include the $17 \mu \mathrm{m}$ feature attributed to PAHs.

- The Draine \& Li (2007) model, also called silicate-graphitePAH model, that assumes a mixture of carbonaceous and amorphous silicates grains, including different amounts of PAH material.

1 See https://www.ias.u-psud.fr/DUSTEM/ for the fortran code and http://dustemwrap.irap.omp.eu/ for the IDL wrapper. 
- The Compiègne et al. (2011) model that comprises PAHs, small $(a<10 \mathrm{~nm})$ and large $(a>10 \mathrm{~nm})$ amorphous carbons (SamC and LamC), and large amorphous silicates (aSil).

- The THEMIS model (Jones et al. 2013) that provides an evolutionary scenario for the dust evolution in the ISM. This model comprises two dust components: a population of carbonaceous grains of amorphous and aliphatic nature $(\mathrm{a}-\mathrm{C}(\mathrm{:H}))$ for the large grains $(a>20 \mathrm{~nm})$ and of more aromatic nature $(\mathrm{a}-\mathrm{C})$ for the smallest ones $(a<20 \mathrm{~nm})$, and a population of large amorphous silicate grains containing nanometer scale inclusions of $\mathrm{FeS}$ (large a-Sil). These two grain populations are covered by an aromatic mantle in the diffuse ISM and in photodissociation regions (PDRs). In dense regions, an additional mantle of more or less aliphatic matter is accreted on top of the aromatic mantle.

Depending on the model, the mid-infrared (MIR) emission $(20-60 \mu \mathrm{m})$ is mainly dominated by VSGs (DBP90), graphites (DL07), and SamC (MC11). With AJ13, the amorphous carbons (a-C), that also include PAHs, has a continuous dust-size distribution that reproduces the MIR emission, and can in some cases dominate the FIR emission if adopting a different power law in the size distribution as compared to the one used to reproduce the Galactic diffuse ISM.

The FIR-submm emission is described by BGs (DBP90), amorphous silicates (DL07), large amorphous carbons, and large silicates (MC11). With AJ13, by adopting the same dust size distribution as in our Galaxy, the large a-C(:H) and silicate grains dominate the emission at long wavelengths. For simplicity, we adopt a single name for the different dust components of the models: PAHs, VSGs (MIR emission), and BGs (FIR-submm emission).

\subsection{Fitting}

Each SED is modeled using the DustEM Wrapper package. These models were first developed to reproduce the diffuse ISM of our Galaxy. We tested each of these models (with Galactic parameters) on the LMC clouds by only constraining the mass abundances relative to hydrogen of the different dust populations $\left(Y_{i}\right)$, the intensity of the NIR continuum $\left(I_{\text {NIR Cont }}\right)$, and the intensity of the radiation field $\left(X_{\mathrm{ISRF}}\right)$. We consider a single intensity of the radiation field (RF). This assumption seems reasonable since this analysis focuses on molecular clouds which are expected to be "cold", mainly in the atomic and molecular phases. As a consequence, a mixture of RF is not required. The ionized gas phase surrounding the clouds can belong to three different categories: diffuse ionized gas, and typical or bright HII regions. As described in Paradis et al. (2011b), the results obtained assuming a composite RF substantially corroborate those derived with a single RF. However, it appears that a composite RF does not always give a better fit than a single RF, and in particular does not reproduce the $70 \mu \mathrm{m}$ excess evidenced in the ionized phase. For instance, in some cases, modeling with DBP90 including a composite RF exhibits an important lack of emission at $70 \mu \mathrm{m}$, as well as a higher $\chi^{2}$, as compared to a single RF modeling (Paradis et al. 2011b). For low temperatures, the use of a composite RF also engenders higher $\chi^{2}$ (Paradis et al. 2012). For all these reasons, we decided to adopt a single RF.

All the DustEM models that include a separate PAH component, that is, DBP90, DL07, and MC11, offer the possibility to have a mixture of PAHs depending on their ionization degrees. We have tested all models with full neutral PAHs and with both neutral and ionized PAHs. Only DL07 gives significantly better results with a mixture of PAHs, whereas the presence of ionized PAHs in the other models does not improve the quality of the fits. With AJ13, adopting the same dust-size distribution for the a-C component as in our Galaxy does not give satisfactory results (see also Chastenet et al. 2017), it reproduces neither the near-infrared (NIR) nor the MIR emission. Therefore, the slope of the power law in the a-C dust size distribution is left as a free parameter.

A NIR-continuum described by a black body at a temperature of $1000 \mathrm{~K}$ is added to the modeling, as in previous studies (see e.g., Flagey et al. 2006; Bernard et al. 2008; Paradis et al. 2011a). Its origin is unknown but is required in addition to the PAH components in most of the cases to reproduce the NIR data. To summarize, for each model we leave the following free parameters in the fits:

- DBP90 (5 free parameters): $Y_{\mathrm{PAH}}, Y_{\mathrm{VSG}}, Y_{\mathrm{BG}}, X_{\mathrm{ISRF}}$ and $I_{\text {NIR Cont }}$.

- DL07 (7 free parameters): $Y_{\mathrm{PAH}^{0}}, Y_{\mathrm{PAH}^{+}} Y_{\text {graphite }}, Y_{\mathrm{B}_{\text {sil }}}$ for big silicates, $Y_{\mathrm{S}_{\mathrm{sil}}}$ for small silicates, $X_{\mathrm{ISRF}}$ and $I_{\mathrm{NIR} \text { Cont }}$.

- MC11 (6 free parameters): $Y_{\mathrm{PAH}}, Y_{\mathrm{SamC}}$ for small amorphous carbons, $Y_{\mathrm{LamC}}$ for large amorphous carbons, $Y_{\mathrm{aSil}}$ for amorphous silicates, $X_{\mathrm{ISRF}}$ and $I_{\mathrm{NIR}}$ Cont .

- AJ13 (7 free parameters): $Y_{\mathrm{a}-\mathrm{C}}$ for small aromatic carbons, $Y_{\text {Large a-C(:H) }}$ for large aliphatic Carbons, $Y_{\text {Large a-Sil }}$ for large amorphous silicates (including two silicate components: $Y_{\text {Oliv. }}$ for olivine-type and $Y_{\mathrm{Pyr}}$ for pyroxene-type), $\alpha_{\mathrm{a}-\mathrm{C}}$ for the slope of the power-law in the a-C dust size distribution, $X_{\text {ISRF }}$ and $I_{\text {NIR Cont }}$.

Typical SEDs obtained in the phases of two molecular clouds fitted with the models are presented in Figs. 2 and 3.

The lack of data between 24 and $70 \mu \mathrm{m}$ is problematic in the sense that there are no further constraints to characterize the VSG component over this wavelength range. The models have also different predictions in the submm depending on their emissivity spectral index. For instance, both previous figures evidence distinct brightness predictions in the submm at $700 \mu \mathrm{m}$ for the same phase. Additional data in the $\mathrm{mm}$ range would be useful to constrain and improve the existing models. However, today, including $\mathrm{mm}$ data from space instruments in the SED directly impacts the angular resolution which has to be degraded.

\subsection{Cloud selection}

The decomposition of the dust emission does not give satisfactory results in some cases. For instance, some decompositions can lead to negative correlations, that can result from low negative emission in some pixels after foreground subtraction or star removal, or from possible correlation between the gas phases for example. Data at 100 and $160 \mu \mathrm{m}$ are both crucial to derive the dust temperature and to determine the dust abundances. We therefore only consider SEDs with positive values at 100 and $160 \mu \mathrm{m}$ in both the atomic and the molecular phases. This criterion induces the rejection of 90 clouds. The same criterion in the ionized phase leads to the removal of 40 additional clouds. We therefore consider a total of 182 clouds for the atomic and molecular phases and 142 clouds for the ionized phase. From these clouds we also exclude those for which SED fitting results in substantial bad fits. This last selection depends on the model used, and can remove $20 \%$ of the clouds in the case of MC11, whereas it concerns $10 \%$ of the clouds with DL07, 5 and $2 \%$ with AJ13 and DBP90.

\section{Modeling residuals}

Distribution of the modeling residuals compared to observations $\left(\right.$ Residual $\left.=\left(I_{v}^{\text {obs }}-I_{v}^{\text {model }}\right) / I_{v}^{\text {obs }}\right)$ are presented in Figs. 4-7 for 
D. Paradis et al.: Revisiting the dust properties in the molecular clouds of the Large Magellanic Cloud
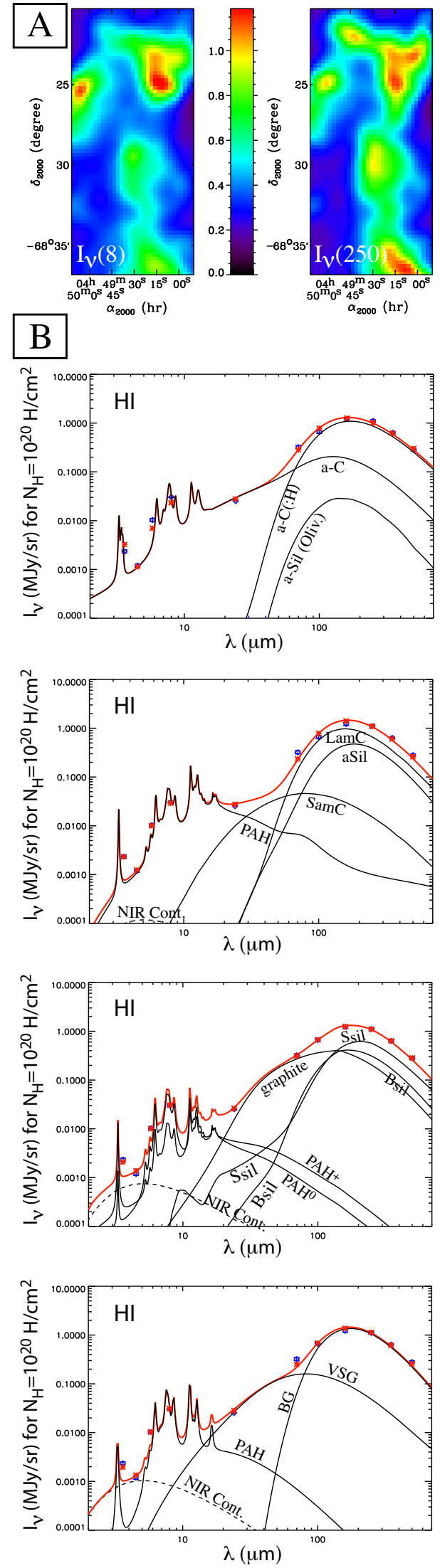
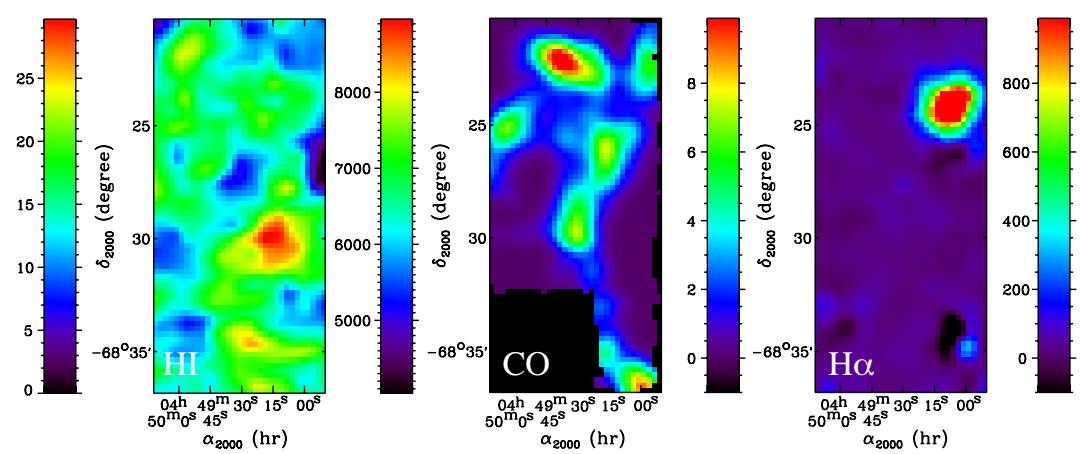

Jones et al. (2013)
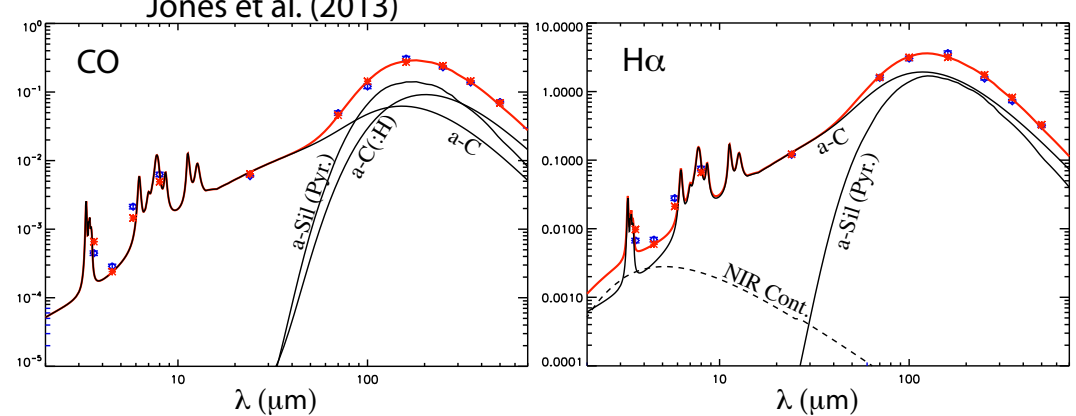

Compiègne et al. (2011)
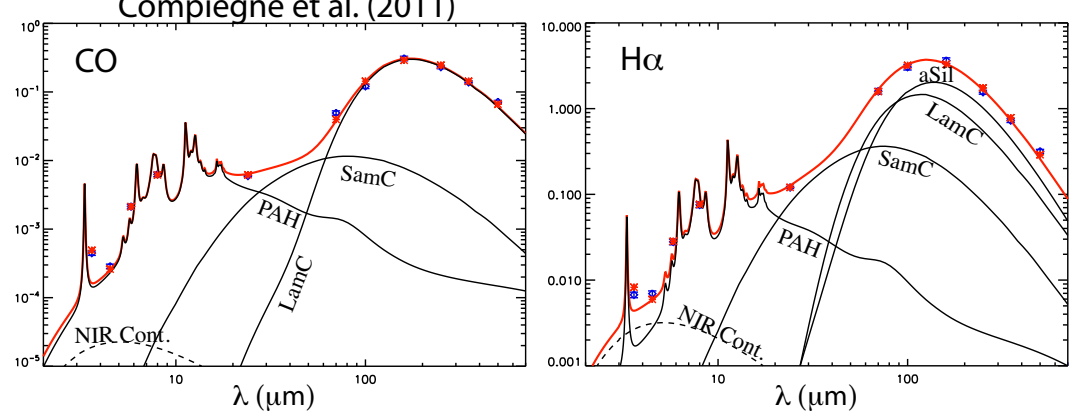

Draine and Li (2007)

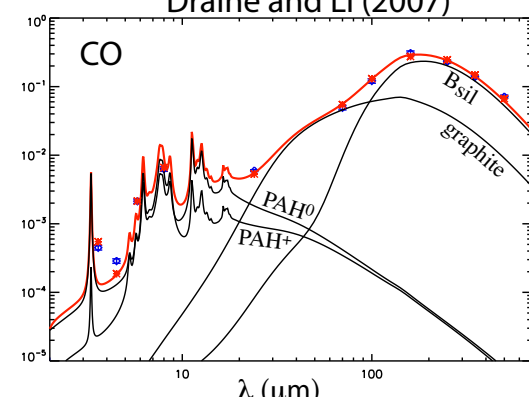

Désert et al. (1990)
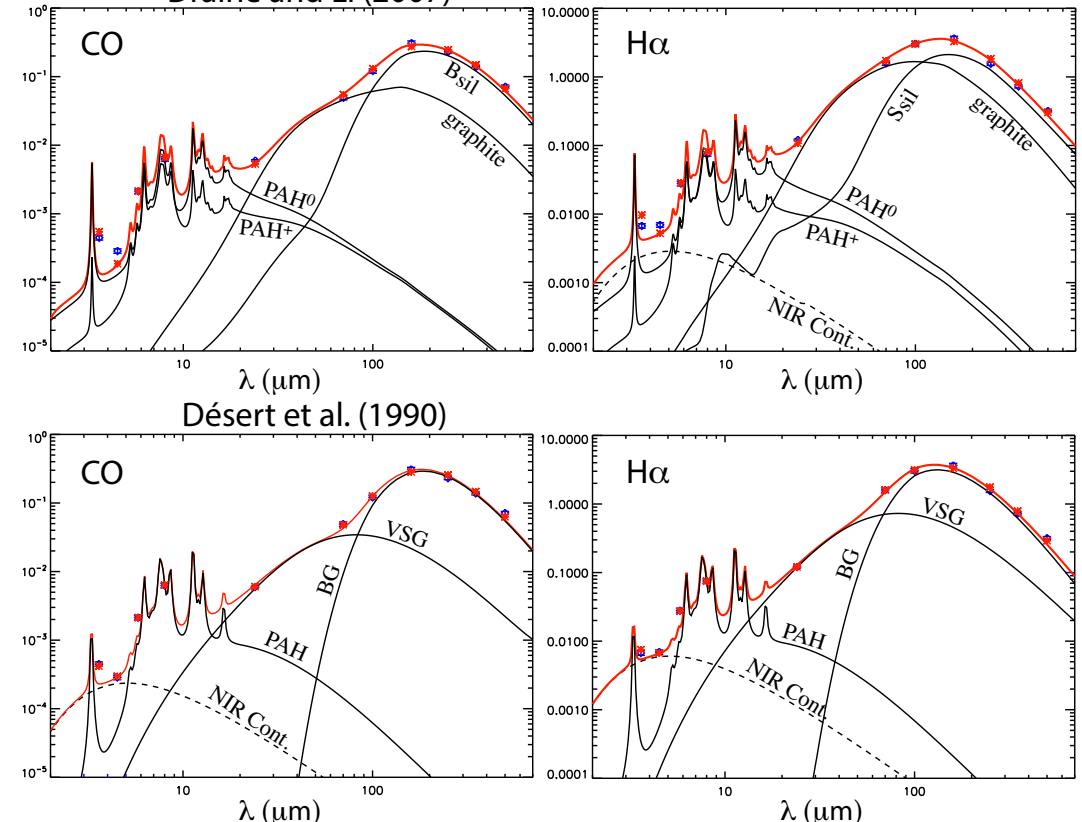

$\lambda(\mu \mathrm{m})$

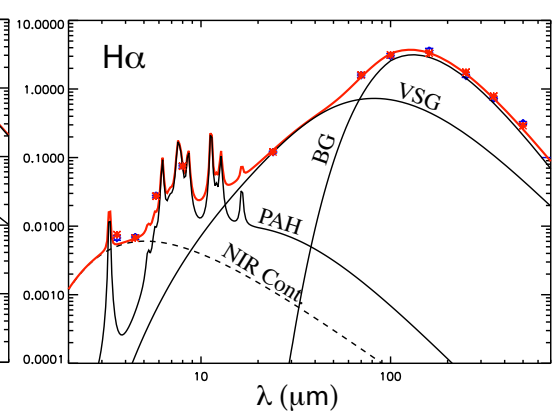

Fig. 2. Panels A: maps of the dust emission ( 8 and $\left.250 \mu \mathrm{m}_{\text {in }} \mathrm{MJy} \mathrm{sr}^{-1}\right)$ and maps of the gas components $\left(\mathrm{HI}\right.$ in Jy beam ${ }^{-1} \mathrm{~km} \mathrm{~s}^{-1}$, CO in $\mathrm{K} \mathrm{km} \mathrm{s}{ }^{-1}$, $\mathrm{H} \alpha$ in dR) of a molecular cloud of our sample (cloud number 5 in Fukui et al. 2008, nomenclature). Panels B: SEDs in the various gas phases derived from dust decomposition (see Eq. (3)) for cloud number 5, fitted with the different dust models. Blue diamonds correspond to the data with their $1 \sigma$ uncertainties, whereas the red asterisks and the red continuous lines correspond to the color-corrected brightnesses and the total emission derived from the models. 

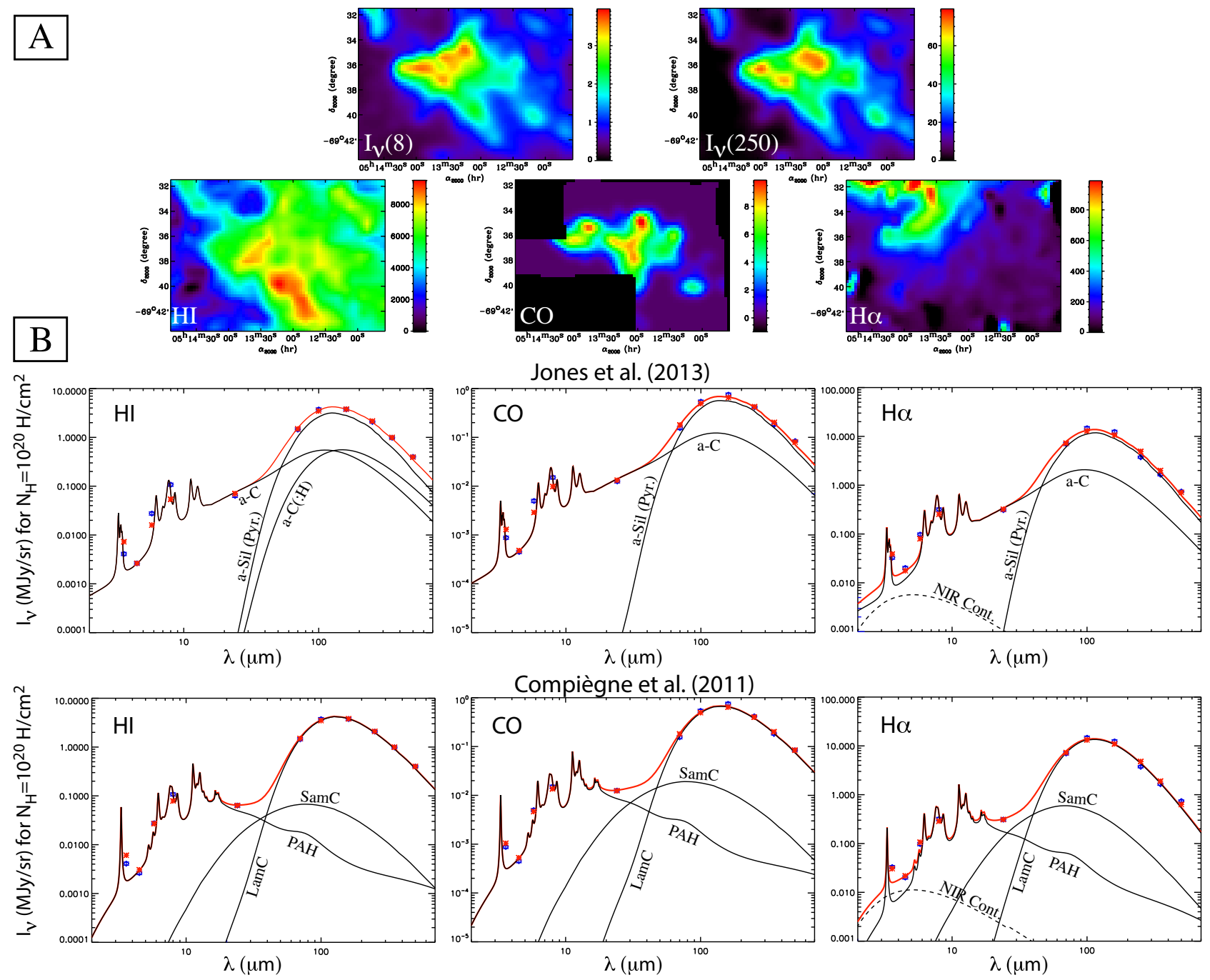

Compiègne et al. (2011)
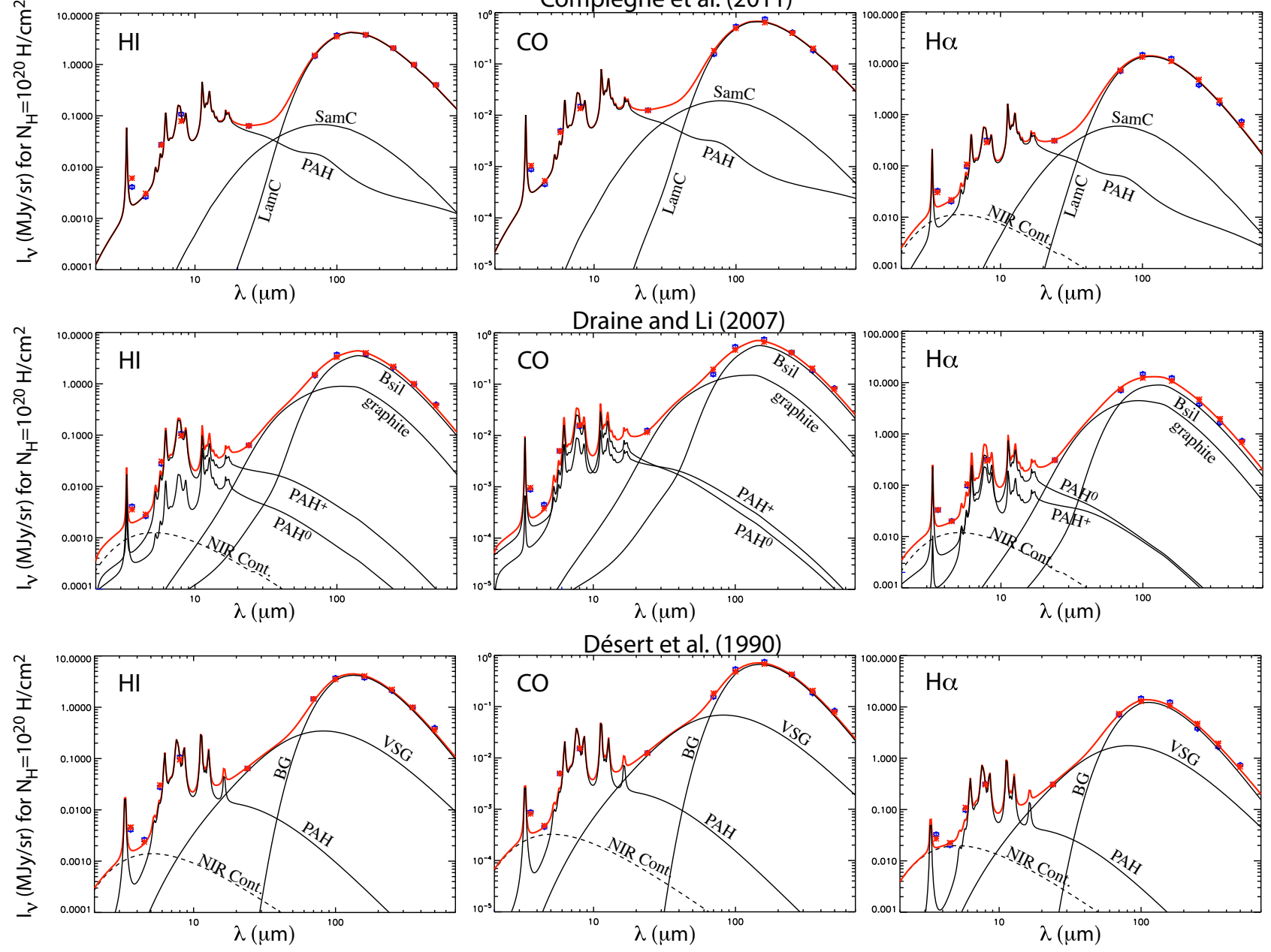

Fig. 3. Panels A: maps of the dust emission ( 8 and $250 \mu \mathrm{m}_{\text {in }} \mathrm{MJy} \mathrm{sr}^{-1}$ ) and maps of the gas components (HI in Jy beam ${ }^{-1} \mathrm{~km} \mathrm{~s}^{-1}$, CO in $\mathrm{K} \mathrm{km} \mathrm{s}^{-1}$, $\mathrm{H} \alpha$ in $\mathrm{dR}$ ) of a molecular cloud of our sample (cloud number 71 in Fukui et al. 2008, nomenclature). Panels B: SEDs in the various gas phases derived from dust decomposition (see Eq. (3)) for cloud number 71, fitted with the different dust models. Blue diamonds correspond to the data with their $1 \sigma$ uncertainties, whereas the red asterisks and the red continuous lines correspond to the color-corrected brightnesses and the total emission derived from the models. 

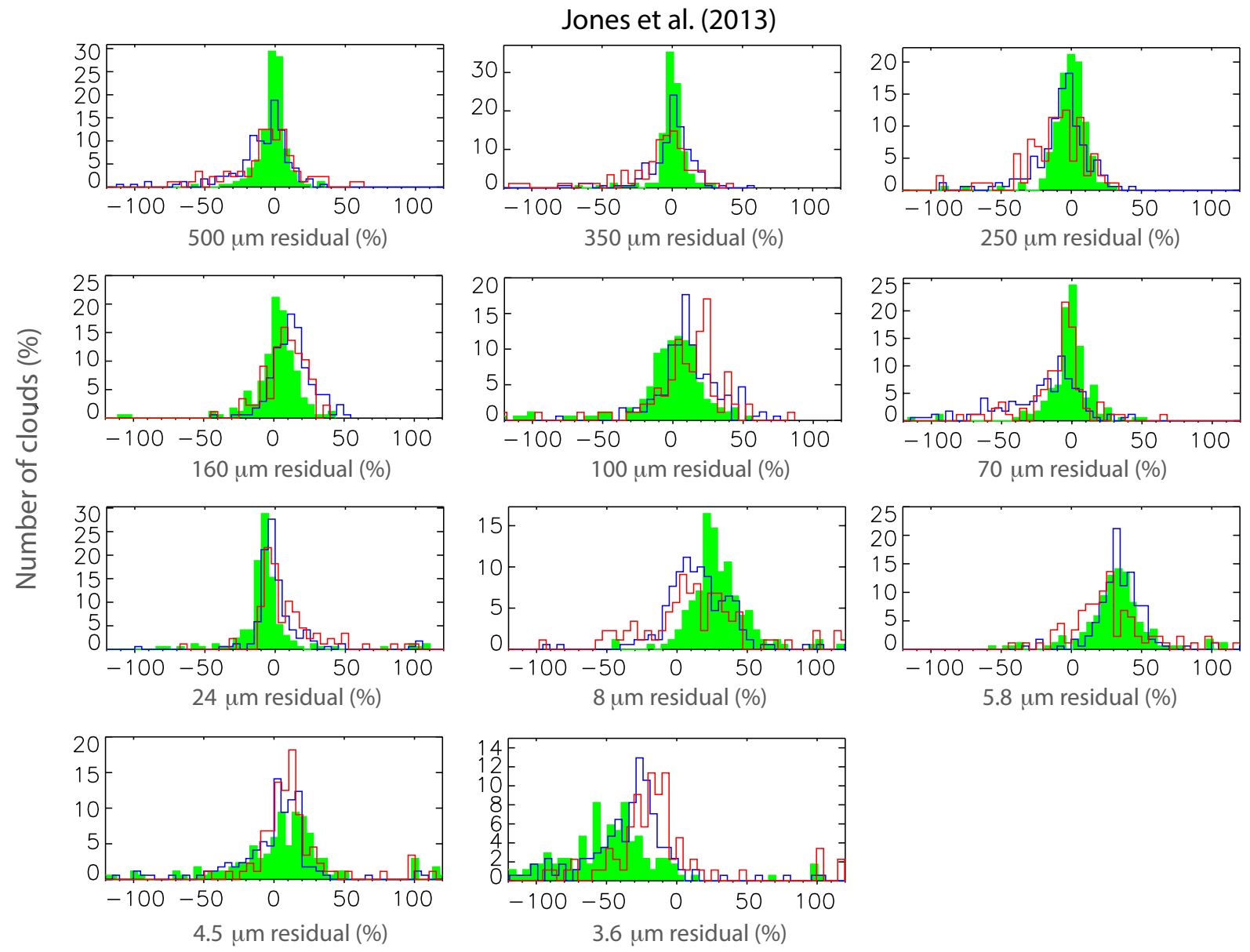

Fig. 4. Histograms of residuals $\left(I_{v}^{\mathrm{obs}}-I_{v}^{\mathrm{model}}\right) / I_{v}^{\mathrm{obs}}$ derived from AJ13, in the atomic (green), molecular (blue), and ionized (red) phases.

each model. We make a Gaussian fit to the residuals with the following function:

$f(x)=A_{o} e^{-\left(\frac{x-A_{1}}{A_{2}}\right)^{2} / 2}$.

The $A_{0}, A_{1}$, and $A_{2}$ parameters correspond to the amplitude, the central value, and the standard deviation of the Gaussian. Values of $A_{1}$ and $A_{2}$ are summarized in Table 1, and $A_{1}$ parameters are plotted in Fig. 8. In this latter figure, we also plot results of the fits obtained with AJ13 when taking the same size distribution of the smallest grains as in our Galaxy, and obtained with DL07 when considering full neutral PAH component for comparison. DL07 clearly requires the mixture of neutral and ionized PAHs to improve the fits between 3.6 and $8 \mu \mathrm{m}$. The $24 \mu \mathrm{m}$ can be reproduced within $15 \%$ by all the models, except with $\mathrm{AJ} 13$ adopting the Galactic size distribution of the a-C component. We clearly see that the models are more or less in agreement with each other within $10 \%$ in the FIR-submm (70-500 $\mu \mathrm{m})$ domain and give reduced residuals compared to the NIR domain. From Table 1, we can see that for wavelengths below $24 \mu \mathrm{m}$ the dispersion between the different results obtained with the models is large and can be as high as $52 \%$ at $3.6 \mu \mathrm{m}, 35 \%$ at 4.5 and $5.8 \mu \mathrm{m}$, and $25 \%$ at $8 \mu \mathrm{m}$. It also shows the highest residuals at 24 and $70 \mu \mathrm{m}$ compared to the other models. AJ13 does not give satisfactory results in the PAH bands with residuals reaching values up to $35 \%$ at $5.8 \mu \mathrm{m}$ and $-47 \%$ at $3.6 \mu \mathrm{m}$ for instance, significantly larger than the calibration uncertainties $(10 \%)$. However, this model has the best description of data at long wavelengths. The main goal to develop this model was to present a global approach to interstellar dust modeling based on laboratory measurements, and taking into account dust evolution in the different gas phases. In addition, this model was configured with the use of Galactic data only, which is probably not optimal when analyzing dust, and in particular the smallest dust particles in the LMC. DBP90 and MC11 are the two best models in this present analysis to reproduce the PAH bands (with a maximal residual of $-14.7 \%$ at $3.6 \mu \mathrm{m}$ ) but DBP90 does not satisfactorily reproduce long-wavelength data. However, this model is the most simple of all the models presented here, and has less free parameters compared to the other models.

\section{Submillimeter flattening}

During recent years, many studies have evidenced a flattening of the spectra in the submm-mm, based on observational data (see for instance Israël et al. 2010; Bot et al. 2010; Galliano et al. 2011; Paradis et al. 2012; Gordon et al. 2014), but also based on laboratory data from grain analogs (Demyk et al. 2017). Whereas several possibilities have been proposed, its origin is still not understood. All the models used here that incorporate a constant emissivity spectral index in the submm-mm domain, that is, all models except AJ13, reveal important absolute variations between residuals at 250 and $500 \mu \mathrm{m}$ in the $\mathrm{HI}$ and $\mathrm{H} \alpha$ phases. This result indicates that the emission spectra derived from the models are systematically steeper compared to the data. The absolute variations of the residuals between the 250 and $500 \mu \mathrm{m}$ are around 7 to $17.5 \%$ in the HI phase, depending on the model. In AJ13, the large carbonaceous grains allow to flatten 

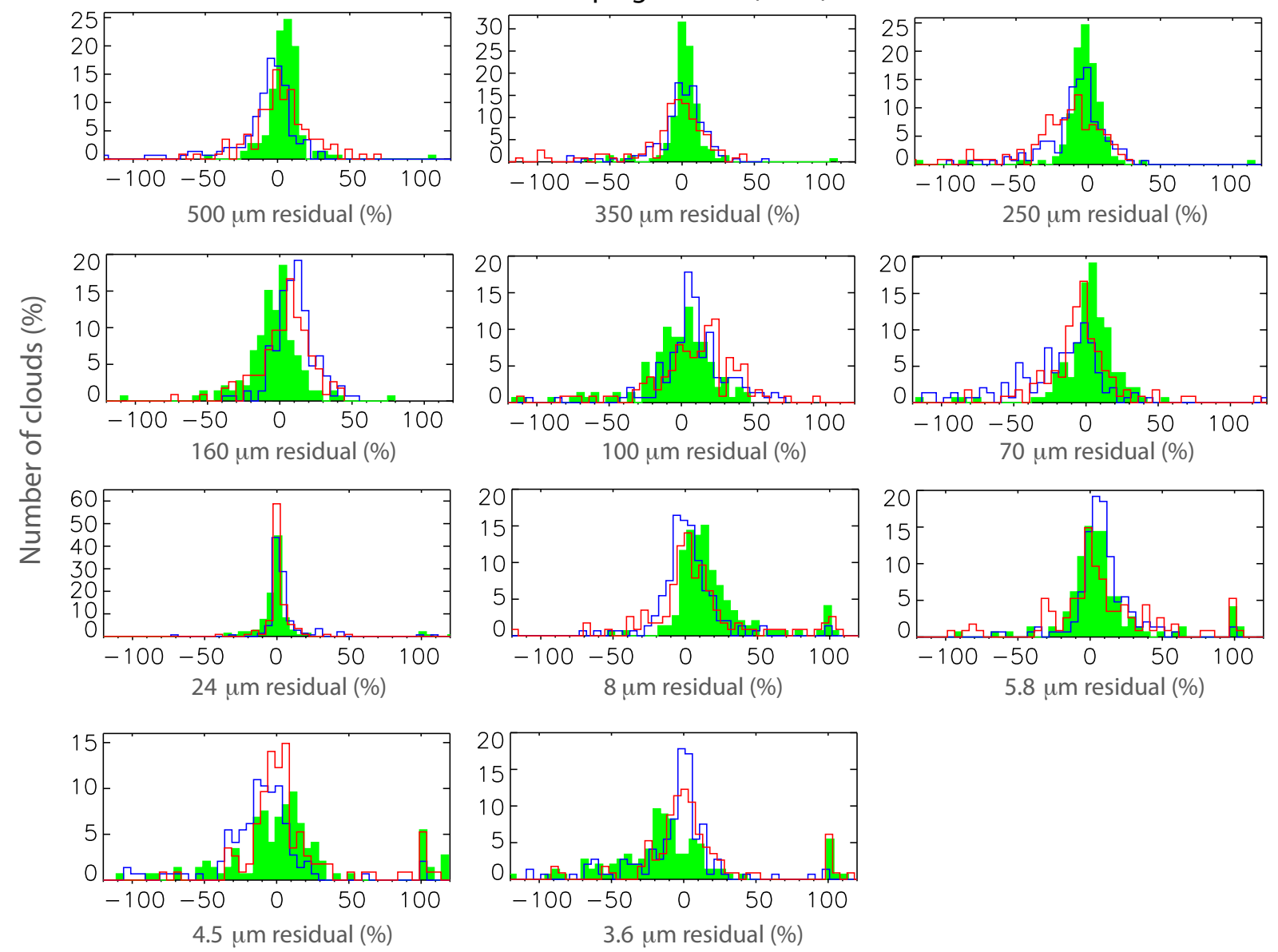

Fig. 5. Histograms of residuals $\left(I_{v}^{\mathrm{obs}}-I_{v}^{\mathrm{model}}\right) / I_{v}^{\mathrm{obs}}$ derived from MC11, in the different gas phases (same colors as in Fig. 4).

the spectra in the submm thanks to the low-emissivity spectral index of this component taken close to one. This is the reason why this model is able to reproduce the data with minimal residual at long wavelengths. As a consequence, the flattening of the spectra in the submm-mm cannot be evidenced by analyzing the residuals with $\mathrm{AJ} 13$. In the $\mathrm{H} \alpha$ phase, residuals between 250 and $500 \mu \mathrm{m}$ also significantly vary in absolute value with a minimum of $\simeq 11.3 \%$ with $\mathrm{MC} 11$ and a maximum of $\simeq 17.6 \%$ with DBP90. This is the first time that evidence has been found of a submm flattening in the ionized gas. However, in the $\mathrm{CO}$ phase the absolute variations are significantly lower, between 0.43 and $6.7 \%$, and within the $7 \%$ calibration uncertainties. These results indicate that statistically dust emission spectra in the molecular phase are steeper than in the $\mathrm{HI}$ and $\mathrm{H} \alpha$ phases. Some studies have highlighted the decrease of the dust emissivity spectral index in the diffuse ISM (Paradis et al. 2012; Planck Collaboration Int. XVII 2014), and we show here for the first time a change in the emissivity spectral index of the various gas phases associated with the molecular clouds. Our results indicate similar behaviors of the dust emission at long wavelengths in the $\mathrm{HI}$ and $\mathrm{H} \alpha$ phases.

We investigated the $500 \mu \mathrm{m}$ residuals as a function of the relative abundances of the different dust components and as a function of the ISRF intensity (see Fig. 9). The comparison of the results obtained with the different dust models does not allow any clear trends or correlations, nor anti-correlations, to be identified between the parameters. Indeed, some trends are clearly model dependent. It is therefore not possible from these findings to evoke any possible links between the $500 \mu \mathrm{m}$ residual and the intensity of the radiation field or the dust abundances.

\section{Dust properties}

\subsection{Intensity of the radiation field and dust temperature}

All models show a statistical decrease of the intensity of the ISRF in the molecular phase as compared to the atomic one (see Fig. 10). In the case of MC11, the decrease of $X_{\text {ISRF }}$ in the molecular phase as compared to the atomic one is not as visible as with the other models, but it exists. Table 2 summarizes the results of Gaussian fits performed on the histograms of the atomic and molecular phases. The distribution for the ionized phase cannot be reproduced by a Gaussian fit. According to the model, the radiation field has a lower intensity in the molecular than in the atomic phase in 61\% (DL07), 63\% (MC11), $83 \%$ (DBP90) or $87 \%$ (AJ13) of the clouds. Conversely, the ionized phase is statistically warmer than the other phases, as visible in the histograms.

The DustEM software considers a size distribution for the different grain component and therefore BGs do not have a single equilibrium temperature. However, we can compute an "approximate" single dust temperature. Indeed, along the line of sight (LOS) we have:

$I_{v} \propto \int_{\mathrm{LOS}} \epsilon N_{\mathrm{H}} Y_{\text {Dust }} X_{\mathrm{ISRF}}$, 
Draine and Li (2007)
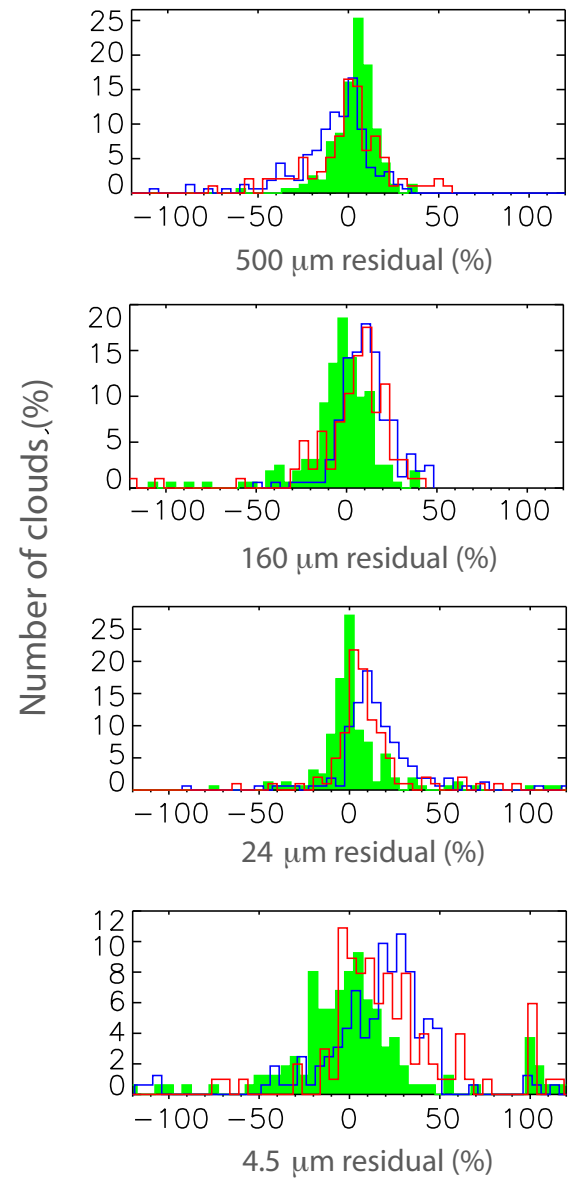
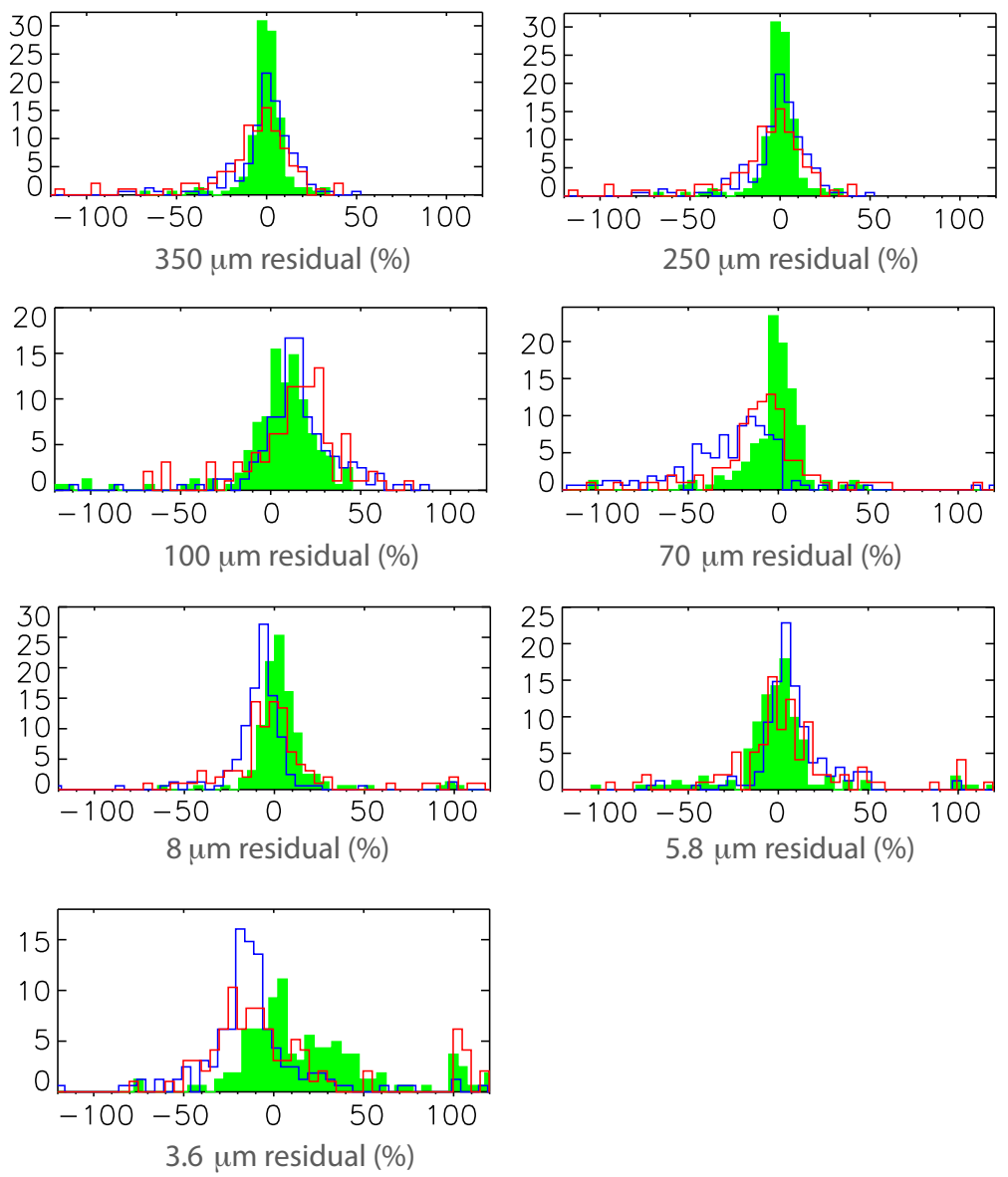

Fig. 6. Histograms of residuals $\left(I_{v}^{\mathrm{obs}}-I_{v}^{\mathrm{model}}\right) / I_{v}^{\mathrm{obs}}$ derived from DL07, in the different gas phases (same colors as in Fig. 4).

where $\epsilon$ is the dust emissivity, $N_{\mathrm{H}}$ the gas column density, $Y_{\text {Dust }}$ the dust abundance, and $X_{\text {ISRF }}$ the intensity of the radiation field. Assuming a gray-body emission with a power-law emissivity, and in the Rayleigh-Jeans limit where $B_{v}(T) \propto 1 / \lambda^{4}$, we obtain the following relation (Paradis et al. 2011a):

$T_{\mathrm{d}}^{\mathrm{LMC}}=\left(X_{\mathrm{ISRF}}^{\mathrm{LMC}}\right)^{1 /(4+\beta)} \times T_{\mathrm{d}}^{\odot}$,

where $\beta$ corresponds to the emissivity spectral index. We assume a dust temperature in the solar neighborhood $\left(T_{\mathrm{d}}^{\odot}\right)$ of $17.5 \mathrm{~K}$ (using $\beta=2$, Boulanger et al. 1996). The emissivity spectral index of our models is fixed and close to a value of two, except with $\mathrm{AJ} 13$ when the large grain component is dominated by carbon grains. However, when looking at Fig. 10, histograms of $Y_{\text {Large a-C(:H) }} / Y_{\text {Dust }}$ and $Y_{\text {Large a-Sil+Large a-C(:H) }} / Y_{\text {Dust }}$ highlight the existence of a significant fraction of clouds with large silicate grains dominating the FIR-submm domain. We know that $\beta$ could vary with wavelength and in particular in the submm-mm domain (Mény et al. 2007; Paradis et al. 2011b; Demyk et al. 2017). However, this behavior is not systematic and has not been observed in some galaxies (see for instance Galametz et al. 2011; Aniano et al. 2012). However, around the peak of BG emission, a range which is crucial to deduce the dust temperature, $\beta$ is usually consistent with a value of two. Adopting a lower spectral index will better fit the dust emission at long wavelength but can bias the determination of the dust temperature. Therefore, assuming a spectral index of two is definitely reasonable. The determination of a single dust temperature in each phase of the gas is useful to distinguish different cases of clouds. We then define four cases of clouds with threshold values that are slightly adjusted from one model to the other to account for almost as many clouds in case 1 as in case 2 :

- Case 1: clouds with similar temperatures in the atomic and molecular phases (with a difference of temperatures $\left|T_{\mathrm{d}}^{\mathrm{HI}}-T_{\mathrm{d}}^{\mathrm{CO}}\right|$ $<T_{\text {tresh,low }}$, with $T_{\text {tresh,low }}=1 \mathrm{~K}$ for AJ13 and DBP90, $0.5 \mathrm{~K}$ for $\mathrm{MC} 11$, and $0.6 \mathrm{~K}$ for DL07).

- Case 2: clouds with significantly colder dust in the molecular phase than in the atomic one (with a difference of temperatures $T_{\mathrm{d}}^{\mathrm{HI}}-\mathrm{T}_{\mathrm{d}}^{\mathrm{CO}}>T_{\text {tresh,high }}$, with $T_{\text {tresh,high }}=4 \mathrm{~K}$ for $\mathrm{AJ} 13$, and $3 \mathrm{~K}$ for the other models).

- Case 3: clouds with colder dust in the molecular phase than in the atomic one (with $T_{\text {tresh,low }}<T_{\mathrm{d}}^{\mathrm{HI}}-T_{\mathrm{d}}^{\mathrm{CO}}<T_{\text {tresh,high }}$ ), excluding clouds in case 2 .

- Case 4: clouds with warmer dust in the molecular phase than in the atomic one (with a difference of temperatures $T_{\mathrm{d}}^{\mathrm{CO}}-T_{\mathrm{d}}^{\mathrm{HI}}>$ $\left.T_{\text {tresh,low }}\right)$.

We obtain:

- With AJ13: $16 \%$ of clouds in both cases 1 and 2, $62 \%$ in case $3,6 \%$ in case 4 .

- With MC11: 19\% of clouds in both cases 1 and 2, 30\% in case $3,32 \%$ in case 4 .

- With DL07: $15 \%$ of clouds in case $1,17 \%$ in case $2,33 \%$ in case $3,35 \%$ in case 4 .

- With DBP90: $24 \%$ of clouds in case $1,21 \%$ in case $2,45 \%$ in case $3,10 \%$ in case 4 . 
Désert et al. (1990)
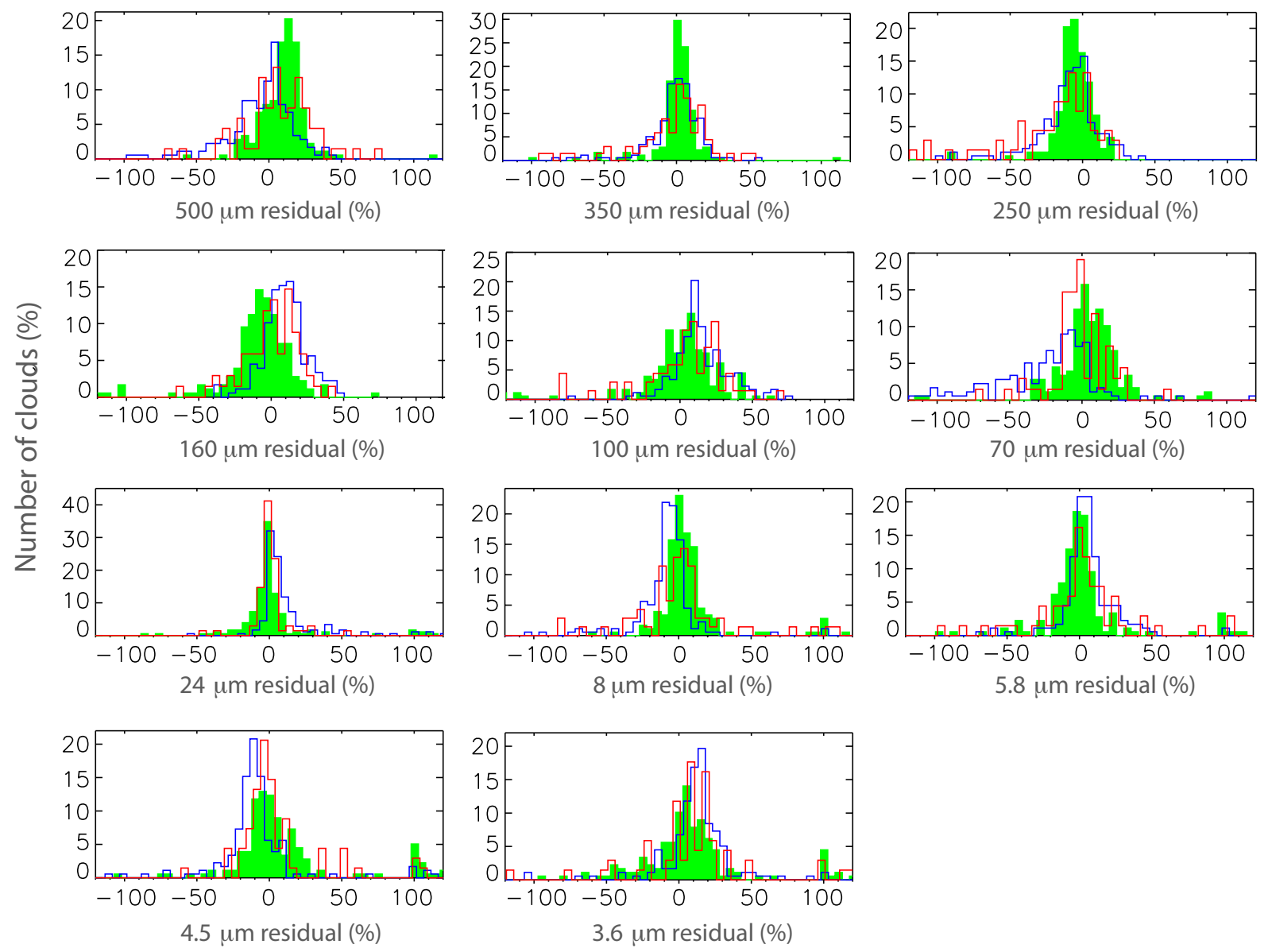

Fig. 7. Histograms of residuals $\left(I_{v}^{\mathrm{obs}}-I_{v}^{\text {model }}\right) / I_{v}^{\mathrm{obs}}$ derived from DBP90, in the different gas phases (same colors as in Fig. 4).

This selection of clouds based on the dust temperature (specifically cases 1 and 2) is used in Sect. 7.2 to analyze the trend of dust abundances in clouds depending on their dust temperatures in the atomic and molecular phases.

\subsection{Relative dust abundances}

Figure 10 shows histograms of the different dust component abundances for each gas phase derived from the models. We can identify an enhancement of the relative abundance of the PAH component (not directly observable with AJ13 since the a-C component is a mixture of small grains and PAHs and is described by a single dust component) in the molecular phase, whatever the model used. This can be visible with a positive shift of the entire histogram or part of it in the molecular phase. The ionized phase tends to show the opposite behavior, that is, a decrease of the PAH relative abundance.

The dust component accounting for the MIR emission (hereafter VSG component) is described by the SamC, graphite, and VSG components according to the models. Histograms in the ionized phase are more extended than histograms in the other phases and mainly show an increase of this VSG component in the ionized gas.

The histogram of the $\mathrm{BG}$ relative abundance is directly linked to the behaviors of the other dust components. Since the BG component accounts for the most important part of the total dust mass (as clearly seen in the histograms), an increase in the $\mathrm{PAH}$ or VGS relative abundance will automatically result in a decrease of the $B G$ relative abundance.
For a comparison, Fig. A.1 presents the histograms of the relative dust abundances when including the dark gas component in the analysis. The previous results remain unchanged.

To go further in our analysis, Fig. 11 compares the dust component abundances in clouds with similar dust temperatures in the atomic and molecular phases (case 1 as defined in Sect. 7.1), and in clouds with significant cold dust in the molecular phase (case 2). For clouds of case 1, one trend can be seen: an increase of the VSG relative abundance in the ionized phase. Concerning the PAHs for clouds in case 1, results cannot be interpreted since they are different depending on the model. When looking at clouds in case 2 , we observe two significant behaviors: an increase of the PAH relative abundance in the molecular phase and an increase of the relative abundances of the VSG component in the ionized phase. The apparent decrease of the $\mathrm{BG}$ relative abundance in the molecular and ionized phase (except with AJ13) only results from the increase of the PAH and VGS relative abundances in these phases. The enhancement of the VSG relative abundance in the ionized phase of clouds is observed in both cases 1 and 2, and therefore does not seem to be related to the dust temperature in the gas phases. The inclusion of the dark gas component in the analysis does not change any of these results (see Fig. A.2).

\subsection{NIR continuum}

The origin of the NIR continuum is unclear but this "component" is required in most cases to explain the dust emission spectra in the NIR, where the PAH component alone fails. Table 3 presents 
Table 1. Central value $\left(A_{1}\right)$ and standard deviation $\left(A_{2}\right)$ of Gaussian fits $\left(f(x)=A_{o} e^{-\left(\frac{x-A_{1}}{A_{2}}\right)^{2} / 2}\right)$ performed on the histograms of the model residuals $\left(I_{v}^{\text {obs }}-I_{v}^{\text {model }}\right) / I_{v}^{\text {obs }}$ in percent (see Figs. $\left.4-7\right)$ for each phase of the gas.

\begin{tabular}{|c|c|c|c|c|c|c|c|c|c|c|c|c|}
\hline \multicolumn{13}{|c|}{ Jones et al. (2013) } \\
\hline & & $3.6 \mu \mathrm{m}$ & $4.5 \mu \mathrm{m}$ & $5.8 \mu \mathrm{m}$ & $8 \mu \mathrm{m}$ & $24 \mu \mathrm{m}$ & $70 \mu \mathrm{m}$ & $100 \mu \mathrm{m}$ & $160 \mu \mathrm{m}$ & $250 \mu \mathrm{m}$ & $350 \mu \mathrm{m}$ & $500 \mu \mathrm{m}$ \\
\hline \multirow[t]{2}{*}{$\mathrm{HI}$} & $A_{1}$ & -47.74 & 10.29 & 31.49 & 25.21 & -7.18 & -2.76 & 1.43 & 2.72 & -0.96 & 0.56 & 0.01 \\
\hline & $A_{2}$ & 26.58 & 18.56 & 12.39 & 13.73 & 5.27 & 6.47 & 14.59 & 9.01 & 8.88 & 5.16 & 5.49 \\
\hline \multirow[t]{2}{*}{$\mathrm{CO}$} & $A_{1}$ & -29.88 & 5.93 & 35.86 & 13.96 & -5.61 & -13.67 & 8.66 & 11.12 & -5.52 & 1.53 & -5.15 \\
\hline & $A_{2}$ & 15.56 & 11.92 & 11.62 & 19.22 & 5.79 & 17.61 & 13.02 & 11.78 & 11.01 & 8.95 & 12.53 \\
\hline \multirow[t]{3}{*}{$\mathrm{H} \alpha$} & $A_{1}$ & -19.56 & 6.94 & 21.24 & 10.38 & -3.67 & -6.57 & 14.83 & 12.53 & -10.16 & -2.19 & -3.98 \\
\hline & $A_{2}$ & 14.20 & 10.61 & 17.54 & 23.39 & 8.45 & 7.70 & 16.83 & 12.90 & 17.59 & 11.85 & 12.28 \\
\hline & \multicolumn{12}{|c|}{ Compiègne et al. (2011) } \\
\hline \multirow[t]{2}{*}{ HI } & $A_{1}$ & -14.74 & 3.55 & 0.93 & 8.10 & -1.56 & 5.56 & 2.40 & -3.48 & -3.52 & 1.33 & 3.68 \\
\hline & $A_{2}$ & 19.55 & 17.00 & 10.36 & 11.59 & 3.25 & 10.45 & 16.82 & 11.54 & 7.79 & 5.65 & 6.99 \\
\hline \multirow[t]{2}{*}{$\mathrm{CO}$} & $A_{1}$ & -1.62 & -9.34 & 7.01 & -1.22 & -1.72 & -12.04 & 9.28 & 7.35 & -6.23 & 1.27 & -4.00 \\
\hline & $A_{2}$ & 7.92 & 14.95 & 8.59 & 11.07 & 3.32 & 18.87 & 12.04 & 10.66 & 10.99 & 10.55 & 9.43 \\
\hline \multirow[t]{2}{*}{$\mathrm{H} \alpha$} & $A_{1}$ & -1.98 & -2.03 & -0.58 & 0.80 & -1.55 & -5.62 & 11.65 & 5.56 & -11.17 & 1.77 & -0.144 \\
\hline & $A_{2}$ & 12.82 & 10.50 & 13.26 & 11.79 & 2.67 & 11.38 & 21.88 & 13.73 & 19.34 & 12.22 & 13.90 \\
\hline \multicolumn{13}{|c|}{ Draine \& Li (2007) } \\
\hline \multirow[t]{2}{*}{ HI } & $A_{1}$ & 5.41 & -1.94 & -2.08 & 0.74 & -1.85 & 0.27 & 9.07 & -2.14 & -5.96 & -2.05 & 4.33 \\
\hline & $A_{2}$ & 24.05 & 19.16 & 9.84 & 7.43 & 5.79 & 7.60 & 13.27 & 10.93 & 7.73 & 5.42 & 8.08 \\
\hline \multirow[t]{2}{*}{$\mathrm{CO}$} & $A_{1}$ & -16.29 & 21.83 & 3.69 & -6.81 & 9.01 & -22.00 & 12.10 & 9.58 & -5.77 & 2.32 & -5.34 \\
\hline & $A_{2}$ & 9.86 & 18.71 & 7.82 & 7.37 & 10.34 & 18.47 & 11.32 & 11.33 & 6.24 & 9.35 & 13.13 \\
\hline \multirow[t]{3}{*}{$\mathrm{H} \alpha$} & $A_{1}$ & -15.27 & 10.02 & 0.98 & -3.55 & 5.25 & -10.38 & 18.32 & 8.09 & -8.23 & -0.15 & 4.55 \\
\hline & $A_{2}$ & 20.87 & 18.89 & 13.70 & 10.78 & 8.19 & 12.20 & 15.27 & 12.29 & 16.54 & 12.91 & 10.70 \\
\hline & \multicolumn{12}{|c|}{ Désert et al. (1990) } \\
\hline \multirow[t]{2}{*}{ HI } & $A_{1}$ & 4.87 & -3.72 & -3.45 & 0.14 & -2.72 & 6.26 & 3.87 & -5.62 & -7.22 & 0.29 & 10.38 \\
\hline & $A_{2}$ & 13.15 & 12.27 & 8.41 & 7.98 & 4.25 & 13.07 & 15.07 & 13.24 & 8.71 & 5.87 & 9.56 \\
\hline \multirow[t]{2}{*}{$\mathrm{CO}$} & $A_{1}$ & 12.45 & -12.84 & 4.10 & -7.66 & 0.83 & -14.99 & 10.05 & 8.71 & -7.12 & 1.96 & -0.45 \\
\hline & $A_{2}$ & 9.02 & 7.53 & 7.42 & 7.19 & 4.86 & 17.35 & 11.46 & 12.31 & 12.66 & 10.75 & 14.99 \\
\hline \multirow[t]{2}{*}{$\mathrm{H} \alpha$} & $A_{1}$ & 8.96 & -5.68 & 1.30 & 1.76 & -2.55 & -5.85 & 9.41 & 1.81 & -8.01 & 3.21 & 9.55 \\
\hline & $A_{2}$ & 12.06 & 8.65 & 12.13 & 10.20 & 3.67 & 8.63 & 17.46 & 15.88 & 16.88 & 14.30 & 18.92 \\
\hline
\end{tabular}

the percentage of SEDs that require a NIR continuum, whatever its intensity. AJ13 shows low percentages in the $\mathrm{HI}$ and $\mathrm{CO}$ phases but is also not able to fully reproduce the NIR observations. The distributions of the intensity of this component over the total dust abundance are presented in Fig. 10. The distribution in the ionized phase is more extended than the ones in the atomic and molecular phases. High values of the NIR continuum are required in the ionized phase to reproduce the SEDs in the NIR, whereas the PAH relative abundance is also reduced in this phase (see Sect. 7.2). If the PAHs were the continuum carriers then we would expect to see variations of these two quantities going in the same directions, and not in the opposite way as observed in our study. Flagey et al. (2006) analyzed the Galactic diffuse ISM in the near-to-mid IR domain and concluded that neither scattered light nor PAH or VSG fluorescence could be responsible of this continuum.

We looked for possible correlations between the continuum and the relative abundance of the different dust components, and no direct link was found. However, the NIR continuum normalized to the total dust abundance seems to be connected to the intensity of the radiation field, as visible in Fig. 12, and this is confirmed by correlation coefficients such as Pearson (going from 0.22 to 0.41 depending on the model) and Spearman (going from 0.20 to 0.43 ). The $p$-values associated to each of the correlations are very low (see Fig. 12) indicating that the correlation is likely to be real. The origin of this continuum is further discussed in Sect. 8.4.

\section{Discussion}

Paradis et al. (2011b) made the first study of dust in the ionized medium of the LMC at $4^{\prime}$ angular resolution. This analysis was done at the Galaxy scale in three regimes of the ionized gas: diffuse, typical and bright HII regions. This work highlighted several results: a decrease of the PAH relative abundance in the ionized phase, as well as an increase of the VSG relative abundance in the ionized phase of bright HII regions compared to typical ones. This phase also showed an enhancement of the NIR continuum. On the other hand, the molecular phase seems to allow the survival of the PAHs. This study was done using the updated DBP90 model. We obtain the same results in this present analysis, at small scale in the molecular clouds, with a larger 

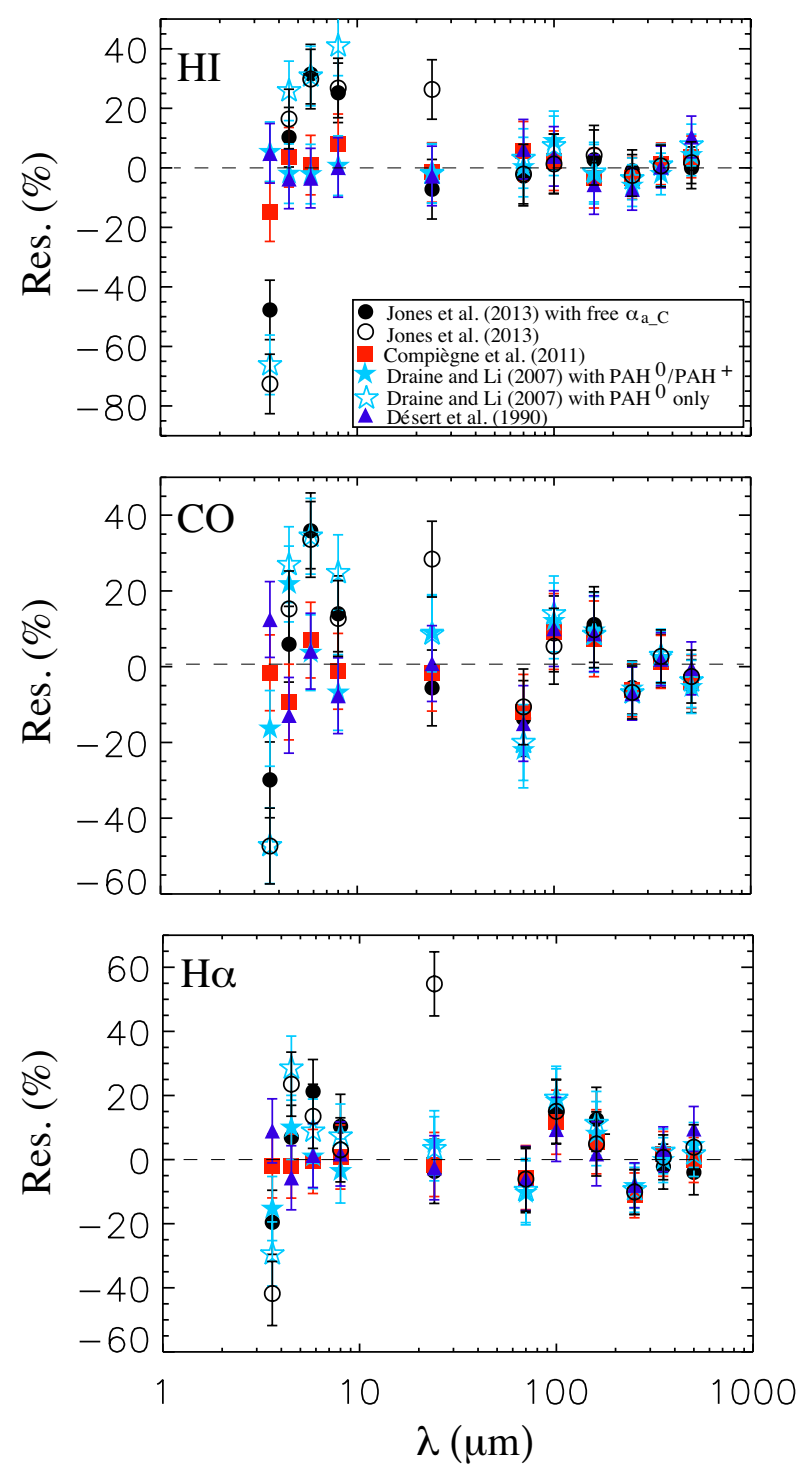

Fig. 8. Average residuals $A_{1}$ based on Gaussian fits $(f(x)=$ $\left.A_{o} e^{-\left(\frac{x-A_{1}}{A_{2}}\right)^{2} / 2}\right)$ of histograms presented in Figs. 4-7 and summarized in Table 1, as a function of wavelength. Error bars correspond to the data calibration uncertainties (10\% for Spitzer and Herschel/PACS data, and $7 \%$ for Herschel/SPIRE data). The dashed line shows the absence of residual $(0 \%)$.

spectral coverage, a better angular resolution, and with the use of four distinct dust models to avoid conclusions that could be model-dependant. We also showed a link between the NIR continuum and the intensity of the radiation field, as well as some variations linked to the $\mathrm{BG}$ component (dust temperature and emissivity slope) according to the gas phases. On the contrary, Paradis et al. (2011a) concluded that statistically, in the majority of the molecular clouds of the LMC, there was no apparent evolution in the PAH and VSG properties between the atomic and the molecular phase at $4^{\prime}$ angular resolution. The origin of this disagreement has been explored and is discussed in the following section.

\subsection{Comparison with Paradis et al. (2011a)}

In Paradis et al. (2011a), we did not observe any changes in the dust component properties between the atomic and molecular phases of the molecular clouds. However, in this present work the method is different for four reasons:

(1) The angular resolution is higher, due to the $1^{\prime}$ resolution of the Mopra instead of the $4^{\prime}$ NANTEN data.

(2) The ionized gas phase associated with the molecular clouds is included in this analysis and was not in Paradis et al. (2011a).

(3) The dark gas is not taken into account (as opposed to Paradis et al. 2011a), but we have checked the consistency of the results if it is included in the analysis.

(4) The wavelength range is extended from 160 to $500 \mu \mathrm{m}$.

Paradis et al. (2011a) performed the modeling with DBP90 only. The main difference between the results from Paradis et al. (2011a) and those of the present study concerns the dust temperature and the PAH relative abundance in the atomic and molecular phases. Indeed, Paradis et al. (2011a) did not observe any temperature decrease or any increase of the PAH relative abundance in the molecular phase. The comparison between the two studies is summarized in Table 4. Other results based on the analysis of the ionized phase or on the submm flattening are not discussed here because these topics were not addressed in Paradis et al. (2011a).

To investigate the origin of theses discrepancies, we performed three tests (described below) with DBP90. Table 4 summarizes the tests and their results.

We first probed the impact of the angular resolution on the results (point 1). We reproduced this present analysis at an angular resolution of $4^{\prime}$ using NANTEN instead of Mopra data. We also restricted our modeling to the Désert et al. (1990) model, as in Paradis et al. (2011a). The results indicate a statistical decrease of the dust temperature, as well as an increase of the PAH relative abundance in the molecular phase as compared to the atomic one. Results of this test are therefore identical to results of this present work performed at $1^{\prime}$ angular resolution. The lower angular resolution in the previous analysis performed by Paradis et al. (2011a) does not explain the differences observed during this present study.

Second, we probed the effect of the potential effect of the absence of the ionized phase in the IR decomposition at an angular resolution of $4^{\prime}$ (point 2 combined to point 1), as in Paradis et al. (2011a). We performed the IR decomposition using the atomic and molecular gas phases only. This time we do not observe a statistical decrease of dust temperature in the molecular phase, but we still see an increase of the PAH relative abundance in the clouds with significant colder dust in the molecular than in the atomic phase. This test shows that the absence of the ionized phase in the analysis, as performed in Paradis et al. (2011a), can significantly affect the dust temperature derived in each phase. This potentially results from an impact on the shape of the SEDs in the FIR wavelength range only. However, this test still does not explain the disagreement in the PAH relative abundance.

Third, we probe the effect of the presence of the dark gas phase on the IR decomposition, still working at an angular resolution of $4^{\prime}$ (point 3 combined with points 2 and 1). The gas components therefore include the atomic, molecular, and dark gas phases, as in Paradis et al. (2011a). In this case, we still do not observe the statistical decrease of the dust temperature in the molecular phase, as in the previous test, but this time the increase of the PAH relative abundance in the molecular phase also disappears. We therefore recover results obtained in Paradis et al. (2011a). It is mainly for this reason that we have not performed further tests based only on the wavelength range.

These tests show that the results can be very sensitive to the gas components considered in the IR decomposition, depending on the angular resolution. Indeed, the more pixels there are 

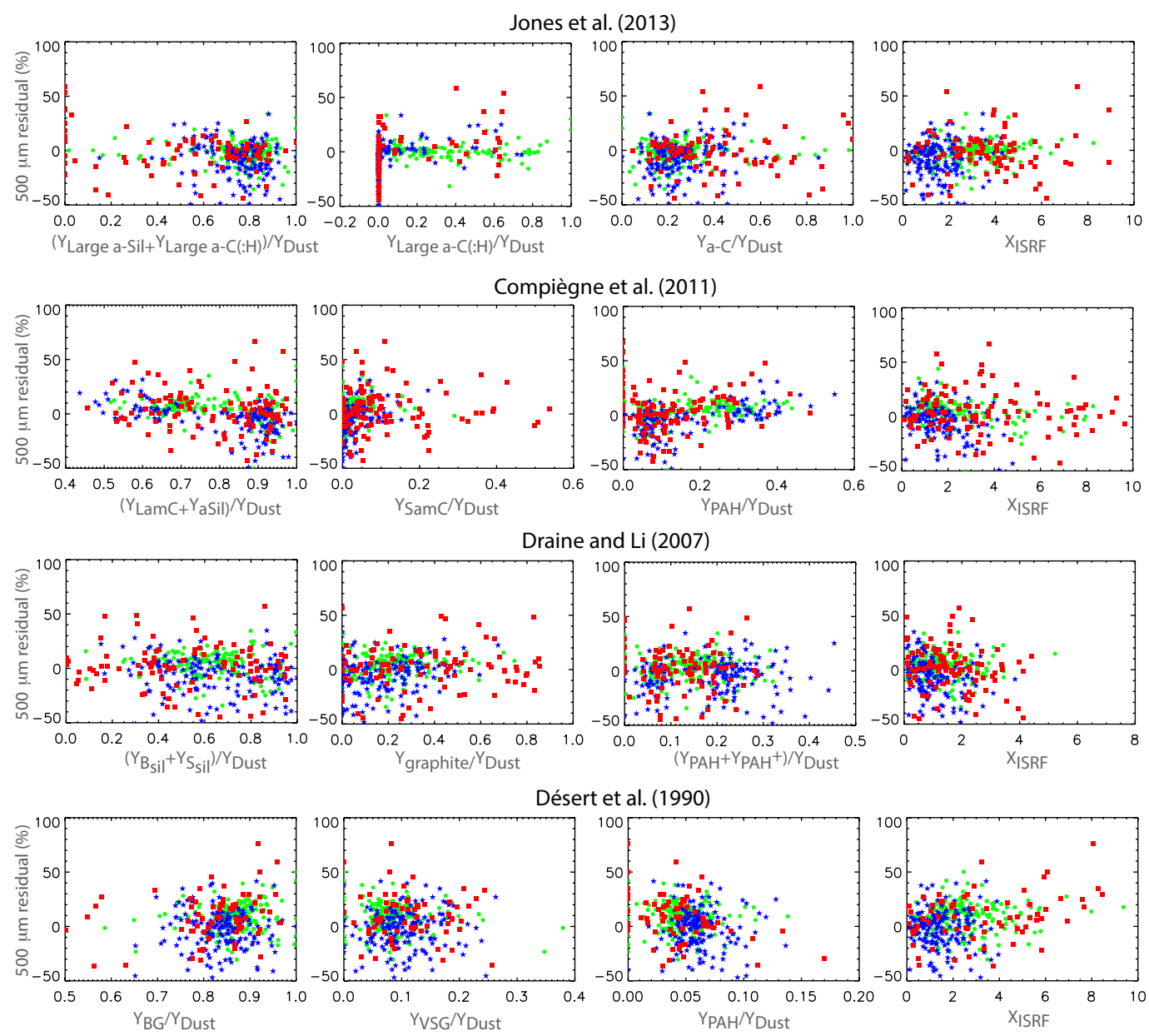

Fig. 9. $500 \mu \mathrm{m}$ residual as a function of the model parameters (relative dust abundances and intensity of the radiation field; see Sect. 4.2) for the different gas phases (atomic in green, molecular in blue, and ionized in red).

in the decomposition, the more the shape of the SED will be unchanged, and the more the results will be unmodified with the presence or not of an additional gas phase. In the present case, the analysis is done at a small scale (molecular cloud scale). Furthermore, the study is sensitive to the number of pixels per cloud and therefore to the angular resolution. For instance, at $1^{\prime}$ resolution, we have seen that whether or not the dark gas is taken into account does not have any impact on the results. However, at $4^{\prime}$ resolution, ignoring the ionized phase has an impact on the dust temperature, and adding the dark gas phase significantly affects results on the PAH relative abundance.

To conclude, the absence of the ionized gas in the dust decomposition in the study performed by Paradis et al. (2011a), combined with the inclusion of the dark gas (highly correlated to the atomic phase), at a lower angular resolution $\left(4^{\prime}\right)$, explains the differences observed between this latter study and the present one.

\subsection{Grain aggregation}

In this present work based on the analysis of molecular clouds at an angular resolution of $1^{\prime}$, we reveal some dust evolution between the atomic and molecular phases:

- most of the molecular clouds show colder dust in the molecular phase than in the atomic one;

- statistically, dust emission spectra in the molecular phase are steeper than in the atomic phase;
- clouds from case 2 (clouds with much colder dust in the molecular than in the atomic phase) point out an increase of the PAH relative abundance.

Furthermore, the ionized phase shows a similar submm flattening to that in the atomic phase, compared to the molecular phase.

Grain aggregation is expected to occur in cold molecular clouds. Big grains could aggregate with one another due to the presence of ice mantles on their surfaces (Stepnik et al. 2003; Kohler et al. 2011, 2012) but could also aggregate VSGs. Here we do not see any signs of VSG disappearance as evidenced in Ysard et al. (2013) and Tibbs et al. (2016). Tibbs et al. (2016) observed a depletion of VSGs $(a<10 \mathrm{~nm})$ in a sample of Galactic cold cores using $1 \mathrm{~cm}$ CARMA observations. They used spinning dust emission observations to constrain the abundance of VSGs and attributed their depletion to grain growth via accretion and coagulation. However, this present study is based on several assumptions and the main one is that the spinning dust hypothesis is the correct interpretation of the anomalous microwave emission. In addition, the study by Tibbs et al. (2016) relies on the analysis of 15 clouds, whereas the sample in our analysis is significantly larger ( $\simeq 170$ clouds). However, our statistical result could describe a general behavior and also hide some specific molecular clouds of the LMC with a decrease of the VGS relative abundance in the molecular phase. Ysard et al. (2013) observed a decrease in the abundance of small carbon grains in a dense filament of the Taurus 

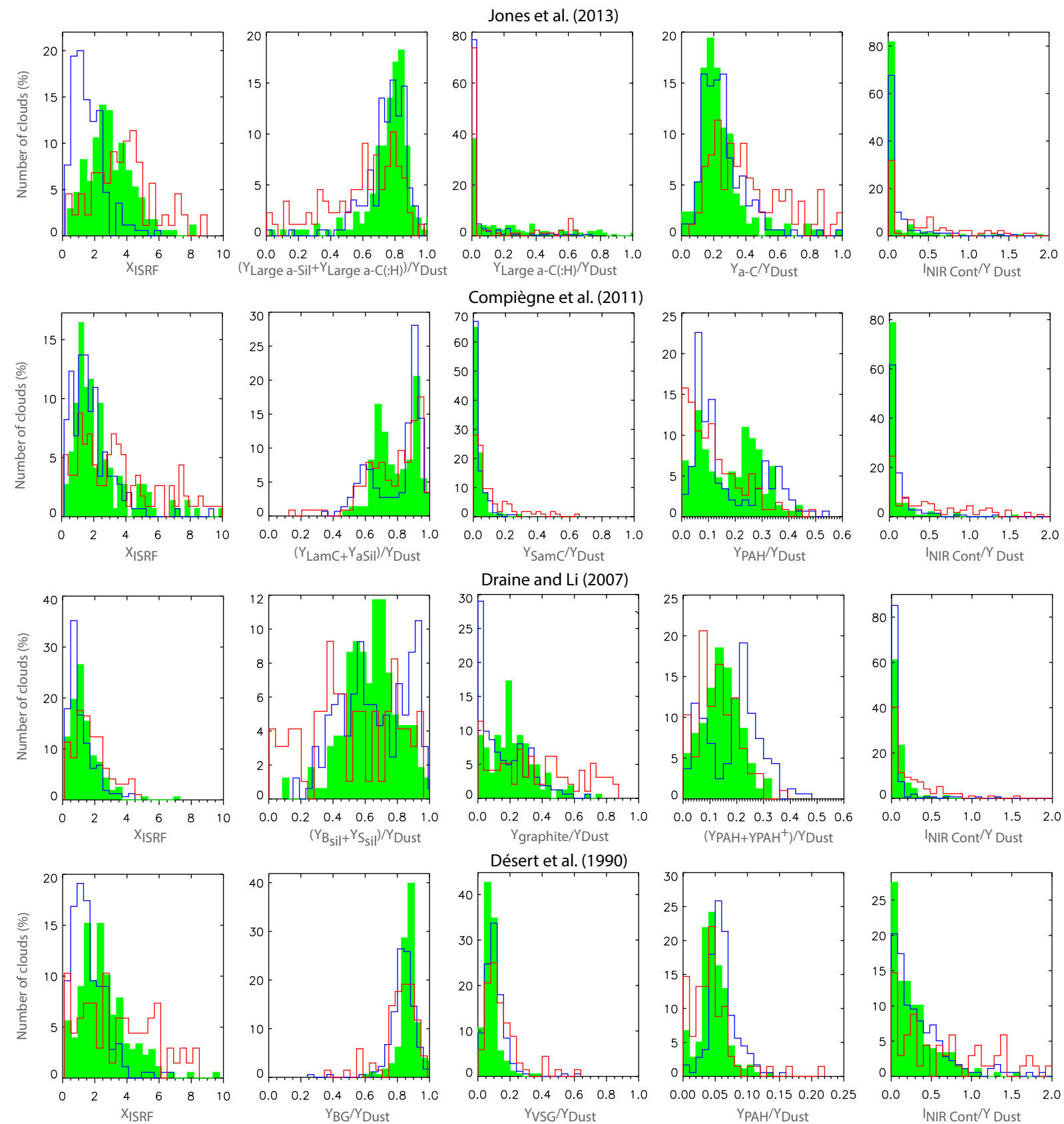

Fig. 10. Histograms of the parameters derived for each dust model (relative dust abundances, intensity of the radiation field, and relative intensity of the NIR continuum; see Sect. 4.2) in the different gas phases (atomic in green, molecular in blue, and ionized in red). We notice a decrease of the intensity of the radiation field in the molecular phase, as well as an increase in the ionized phase, as compared to the atomic phase. We also observe an increase of the PAH relative abundance in the molecular phase (through a positive shift of the entire or part of the histogram in the molecular phase as compared to the atomic phase), and an increase of the VSG relative abundance in the ionized phase. The distribution of the NIR-continuum is more extended in the ionized phase than the ones in the atomic and molecular phases.

Table 2. Central value $\left(A_{1}\right)$ and standard deviation $\left(A_{2}\right)$ of Gaussian fits $\left(f(x)=A_{o} e^{-\left(\frac{x-A_{1}}{A_{2}}\right)^{2} / 2}\right)$ performed on the histograms of $X_{\text {ISRF }}$ for the atomic and molecular phases for each dust model.

\begin{tabular}{lccccc}
\hline \hline & & AJ13 & MC11 & DL07 & DBP90 \\
\hline HI & $A_{1}$ & 3.107 & 1.313 & 1.004 & 1.925 \\
& $A_{2}$ & 1.659 & 0.861 & 0.669 & 1.268 \\
\hline CO & $A_{1}$ & 1.260 & 1.055 & 0.441 & 1.034 \\
& $A_{2}$ & 1.062 & 1.196 & 0.945 & 1.094 \\
\hline
\end{tabular}

molecular complex (L1506) using Herschel data. Their spatial scale allowed them to reach significantly higher densities than what we can probe in this present analysis due to the resolution and the distance of the LMC. Therefore, the difference between the studies could result from a difference in local density, from specific cases that do not apply to most of the molecular clouds, or from different dust properties of the LMC molecular clouds compared to those in our Galaxy.

Big grain coagulation from the diffuse to the dense medium could induce a change in the dust emissivity spectral index between the two phases (Paradis et al. 2009), evidenced here 

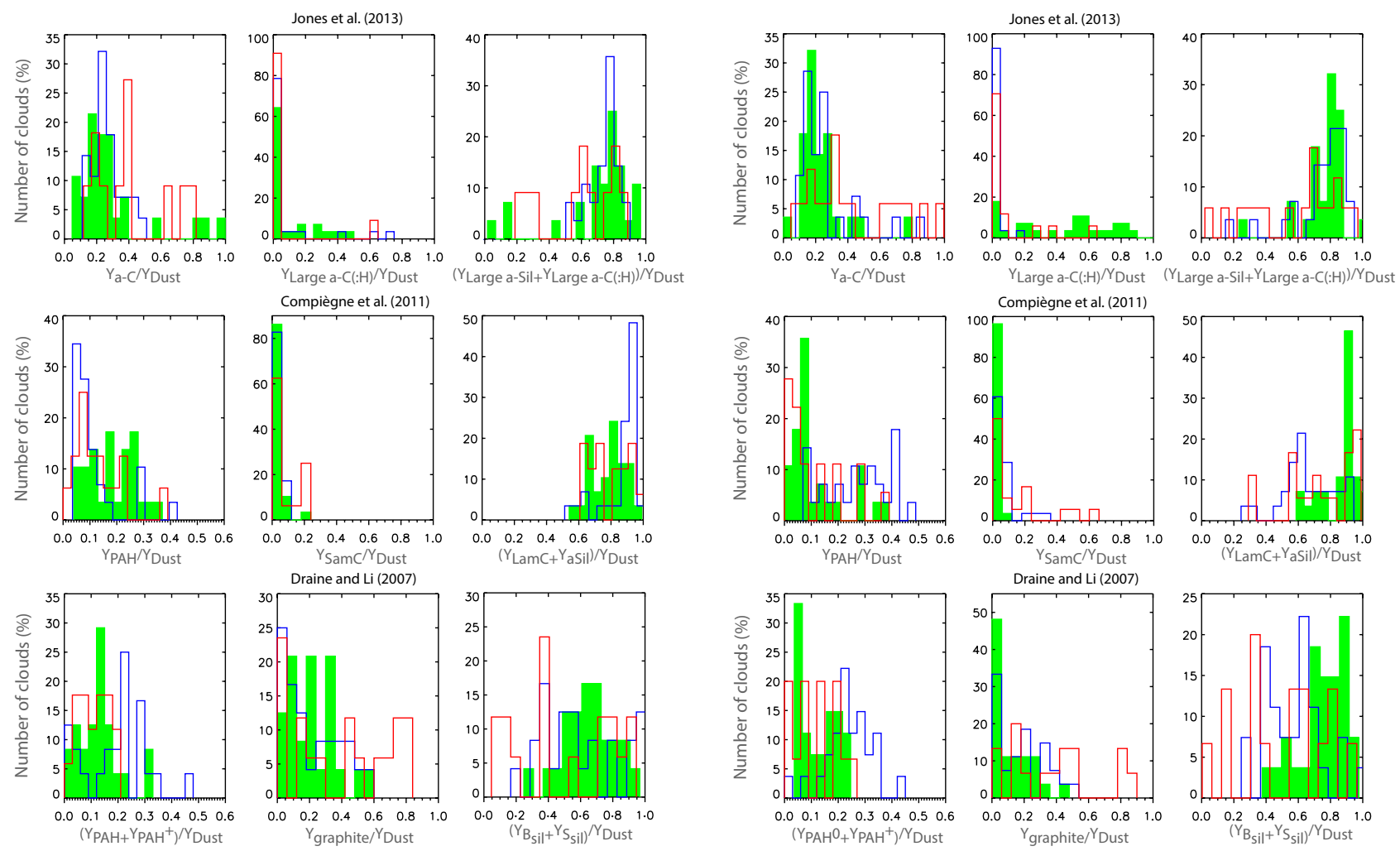

Draine and 1 (2007)
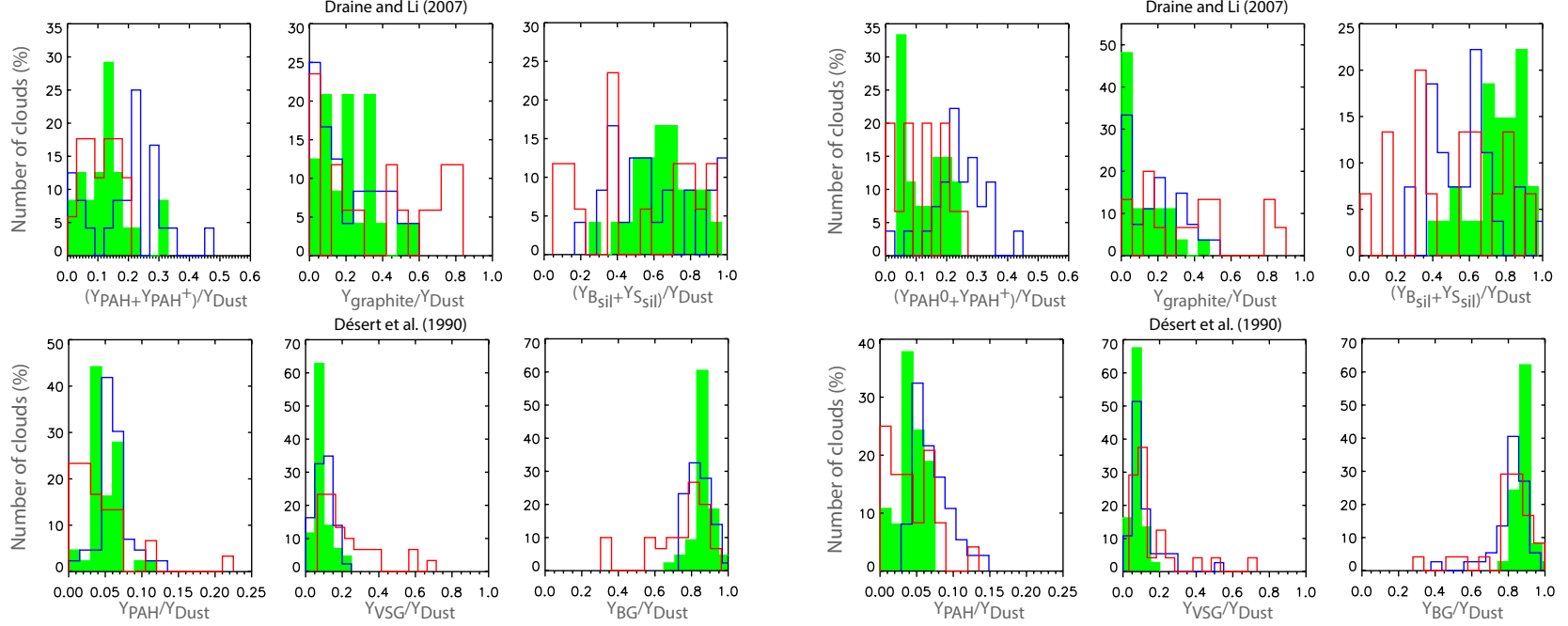

Fig. 11. Histograms of the relative abundances of the dust components for clouds with similar dust temperatures in the atomic and molecular phases (case 1 as defined in Sect. 7.1) on the left, and for clouds with significantly colder dust temperatures in the molecular than in the atomic phases (case 2) on the right. The atomic, molecular, and ionized phases are shown in green, blue, and red, respectively. We clearly evidence a positive shift in the distribution of the PAH relative abundance in the molecular phase for clouds in case 2 as compared to clouds in case 1 . The VSG relative abundance seems to be increased in the ionized phase in both cases.

Table 3. Percentage of SEDs for which the presence of a NIR continuum is required, as a function of the dust emission models.

\begin{tabular}{lcccc}
\hline \hline & AJ13 & MC11 & DL07 & DBP90 \\
\hline HI & 19 & 34 & 69 & 82 \\
CO & 29 & 58 & 37 & 89 \\
H $\alpha$ & 65 & 78 & 81 & 68 \\
\hline
\end{tabular}

by steeper dust emission spectra at submm wavelengths in the molecular phase as compared to the atomic phase.

Jones et al. (2013) proposed a scenario of dust evolution with density, based on the formation of a second mantle of more or less aliphatic matter accreted on top of the aromatic mantle. This model predicts higher spectral index in molecular clouds (because of small particle accretion and a-C:H mantle accretion) than in the diffuse ISM (large homogenous a-C grains). However, because a-C $(: \mathrm{H})$ materials are significantly less emissive than a-C grains, their effects on the FIR-submm emissivity may not be observable. In addition to the second mantle, an ice mantle can be formed at the surface of the grains, favoring grain coagulation. Such a scenario may explain the observed steeper spectra in dense phases relative to the atomic and ionized phases (the second mantle being destroyed by high-intensity radiation fields).

In the framework of the TLS model (Mény et al. 2007), the change in the emissivity spectral index could be the result of variations in the degree of amorphization of the grains, that is, the disorder at atomic and/or nanometer scales (Paradis et al. 2012). Concretely, to explain the FIR-to-submm flattening in the diffuse and ionized phase, the structure of the dust should be different in the atomic and the ionized phase as compared to the molecular phase; it should be more altered, containing more defects or voids for example in the atomic/ionized phase than in the molecular phase. Such an alteration could be the result of dust processing by low-energy cosmic rays. Strong shocks due to supernova explosions could be another alternative to alter dust in the atomic and ionized phases. Moreover, shocks could destroy some of the largest grains and increase the amount of very small grains, as observed in the ionized phase. Supernova explosions would also contribute to the increase of the dust temperature in the ionized phase. 

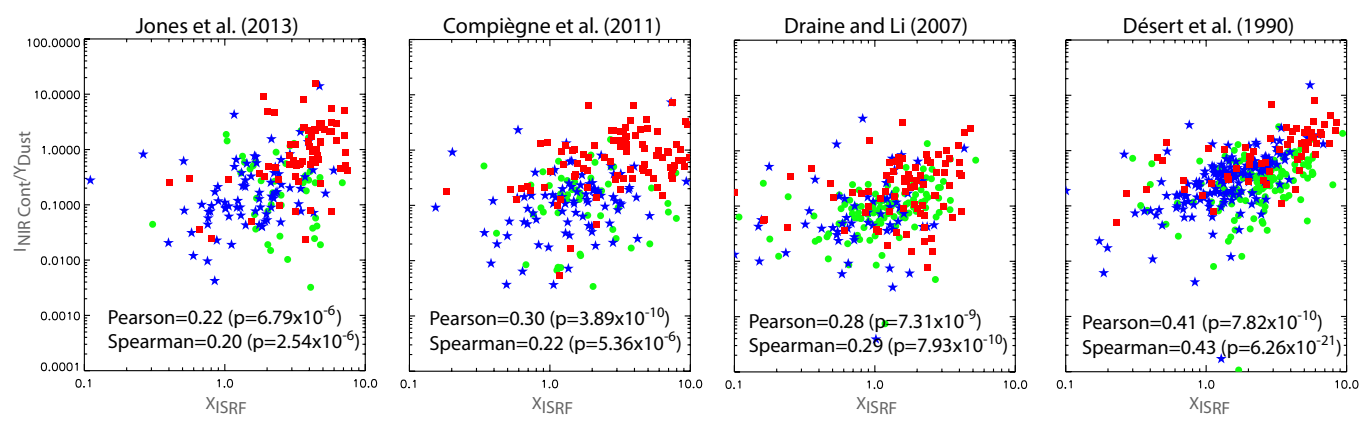

Fig. 12. Intensity of the NIR continuum normalized to the total dust abundance as a function of the intensity of the interstellar radiation field, for the different dust models. The atomic, molecular, and ionized phases are shown in green, blue, and red, respectively. The Pearson and Spearman correlation coefficients are given in each panel.

Table 4. Summary of the method used in this work and Paradis et al. (2011a), and the different tests we performed to allow the comparison of these two studies.

\begin{tabular}{|c|c|c|c|c|}
\hline & This work & Paradis et al. (2011a) & Test & Results of the tests \\
\hline Resolution & $1^{\prime}$ & $4^{\prime}$ & $\begin{array}{l}4^{\prime}, \text { with } \mathrm{HI} / \mathrm{CO} / \mathrm{H} \alpha \\
\text { Model: } \mathrm{DBP} 90\end{array}$ & $T_{\mathrm{d}}^{\mathrm{CO}} \searrow$ and $\left(\frac{Y_{\mathrm{PAH}}}{Y_{\text {Dust }}}\right)^{\mathrm{CO}} \nearrow$ \\
\hline $\mathrm{H} \alpha$ phase & Yes & No & $\begin{array}{l}4^{\prime}, \mathrm{HI} / \mathrm{CO}(\mathrm{H} \alpha \text { removed }) \\
\text { Model: DBP90 }\end{array}$ & $T_{\mathrm{d}}^{\mathrm{CO}} \simeq T_{\mathrm{d}}^{\mathrm{HI}}$ and $\left(\frac{Y_{\mathrm{PAH}}}{Y_{\text {Dust }}}\right)^{\mathrm{CO}} \nearrow$ \\
\hline Dark gas phase & No (yes) & Yes & $\begin{array}{l}4^{\prime}, \mathrm{HI} / \mathrm{CO}+\mathrm{Dark} \text { gas } \\
\text { Model: DBP90 }\end{array}$ & $T_{\mathrm{d}}^{\mathrm{CO}} \simeq T_{\mathrm{d}}^{\mathrm{HI}}$ and $\left(\frac{Y_{\mathrm{PAH}}}{Y_{\text {Dust }}}\right)^{\mathrm{CO}} \simeq\left(\frac{Y_{\mathrm{PAH}}}{Y_{\text {Dust }}}\right)^{\mathrm{HI}}$ \\
\hline $\begin{array}{l}\text { Wavelength range } \\
\text { Modeling }\end{array}$ & $\begin{array}{l}3.6-500 \mu \mathrm{m} \\
\text { AJ13, MC11 } \\
\text { DL07, DBP90 }\end{array}$ & $\begin{array}{l}3.6-160 \mu \mathrm{m} \\
\text { DBP90 }\end{array}$ & - & - \\
\hline Comparison of the results & $\begin{array}{l}T_{\mathrm{d}}^{\mathrm{CO}} \searrow \\
\left(\frac{Y_{\mathrm{PAH}}}{Y_{\text {Dust }}}\right)^{\mathrm{CO}} \nearrow\end{array}$ & $\begin{array}{l}T_{\mathrm{d}}^{\mathrm{CO}} \simeq T_{\mathrm{d}}^{\mathrm{HI}} \\
\left(\frac{Y_{\mathrm{PAH}}}{Y_{\mathrm{BG}}}\right)^{\mathrm{CO}} \simeq\left(\frac{Y_{\mathrm{PAH}}}{Y_{\mathrm{BG}}}\right)^{\mathrm{HI}}\end{array}$ & & \\
\hline
\end{tabular}

Notes. This table does not show all the results of this present analysis, but only results that can be directly comparable with Paradis et al. (2011a). Columns 2 and 3 describe the angular resolution, the presence or not of the ionized and dark gas phases (in addition to the atomic and molecular phases), the wavelength range, the model(s) used, and the results of these two studies in terms of dust temperature and PAH relative abundance in the atomic and molecular phases. Column 4 describes the different tests we applied, indicating the angular resolution, the gas phases we considered in the analysis, and the model we used. Column 5 summarizes the results of the tests.

These two previous scenarios coming from different dust models (AJ13 and TLS) could explain the fact that no link between the submm flattening and the small dust component abundances has been found.

\subsection{Grain formation/destruction}

Polycyclic aromatic hydrocarbons (PAHs) seem to be destroyed in the ionized phase, whereas their relative abundance is enhanced in the cold molecular phase, as observed in Paradis et al. (2011b). The increase of the radiation field in the ionized phase might be responsible for their destruction. Sandstrom et al. (2010) also pointed out a low-mass fraction of PAHs in the diffuse regions of the Small Magellanic Cloud compared to a higher fraction in dense regions. Their study is based on the Draine \& Li (2007) model. It appears therefore that both Magellanic Clouds show an enhancement of the PAH relative abundance in the dense phases. Sandstrom et al. (2012) propose a scenario in which PAHs are formed in regions of dense gas, with a smaller average size, and are more neutral than in galaxies with higher metallicity. This scenario proposes that they then go on to emerge in the diffuse ISM where a fraction of them could be destroyed under typical ISM conditions, since UV photons, shocks, cosmic rays, or hot gas destroy small PAHs more efficiently than large PAHs. Our analysis indicates that the PAH bands in all gas phases are best reproduced by MC11 and DBP90, with full neutral PAHs, but with similar dust size distribution to that in our Galaxy. Our study does not seem to favor the hypothesis of smaller PAHs in the LMC, as compared to the Milky Way for instance. However, our present analysis could also indicate the destruction of PAHs by the higher-intensity radiation field, as PAHs do not disappear in the atomic gas which has typical ISM conditions. Moreover, PAHs could be formed in clouds with significantly colder dust in the molecular phase compared to the atomic phase.

The relative abundance of VSGs is enhanced in the ionized phase, but does not seem to show any link to the nature of the clouds (quiescent or with star formation activity), since their abundances increase in the ionized phase whatever the intensity of the radiation field. Therefore, VSGs could mainly be formed in HII regions rather than in molecular clouds. Since we do not see any signs of VSG coagulation onto BGs, we cannot favor the hypothesis according to which VSGs are released from the surface of BGs to explain the increase of their abundance in the ionized phase. However, another possibility could be the destruction of larger BGs in shocks. 


\subsection{Near-infrared continuum}

In Sect. 7.3 we showed a correlation between the intensity of the NIR continuum and the intensity of the interstellar radiation field. However, no link was found between the continuum and the relative abundances of the different dust components. This continuum was first observed in reflection nebulae (Sellgren et al. 1983), and then in the Galactic ISM (Flagey et al. 2006), but also in high-redshift galaxies (Mentuch et al. 2009). The latter authors detected this continuum in a sample of 88 galaxies from the Gemini Deep Survey. They concluded that circumstellar disks of young massive objects are the most likely candidates to explain the $2-5 \mu \mathrm{m}$ excess. At the Galaxy scale where the total IR luminosity is dominated by massive stars, this hypothesis could be plausible but at small scales where we discern the IR emission coming from the different gas phases, it would be difficult to explain the presence of the NIR continuum in the atomic phase and in the cold molecular phase. In addition, $\mathrm{Lu}$ (2004) showed that the average 3-10 $\mu \mathrm{m}$ spectral shape of the ISM emission does not vary significantly from diffuse sources to star-forming regions.

Duley (2009) investigated the origin of the NIR continuum in the ISM and suggested that carbon molecules and especially dimers of dehydrogenated carbon molecules with less than 28 atoms could be heated to high temperatures $(\simeq 1500 \mathrm{~K})$ by absorption of UV photons. This could explain the link between the intensity of the NIR continuum and the intensity of the interstellar radiation field. Furthermore, this assumption could explain the fact that the continuum appears to unrelated to the relative abundances of the dust components used in the models.

\section{Conclusions}

We performed a decomposition of the IR emission with the different gas phases (atomic, molecular, and ionized phase, as traced by $\mathrm{HI}, \mathrm{CO}$, and $\mathrm{H} \alpha$ emissions, respectively) associated with the LMC molecular clouds. We tested the strength of our conclusions by either taking into account the dark gas or not in the dust decomposition; the conclusions are not affected. The analysis was performed at an angular resolution of 1' using Spitzer and Herschel data for the IR emission, and ATCA/Parkes, Mopra, and SHASSA data for the gas tracers. The SEDs resulting from the correlations were modeled using four different dust models: Jones et al. (2013, AJ13), Compiègne et al. (2011, MC11), Draine \& Li (2007, DL07), and an improved version of Désert et al. (1990, DBP90). Whereas all models give similar fits (within a few percent) between 70 and $500 \mu \mathrm{m}$, the $24 \mu \mathrm{m}$ data require a change in the size distribution of the a-C component in $\mathrm{AJ} 13$, as compared to the Galactic one. The main differences in the model appear in the PAH bands, with the largest residuals being seen in the case of AJ13. DL07 clearly needs a mixture of neutral and ionized PAHs, whereas MC11 and DBP90 give satisfactory results with fully neutral PAHs. This multi-modeling analysis is important to ensure the strength of each of our results presented here.

We found direct evidence for the evolution of dust properties from the atomic to the molecular medium, that manifests through a decrease in the intensity of the radiation field in the molecular phase affecting the dust temperature. Furthermore, we observe a change in the slope of the emission spectrum in the molecular phase, and in particular a steeper spectrum in the FIR to submm range. These results could be the consequence of $\mathrm{BG}$ coagulation in the dense phase. In addition, the cold molecular phase tends to favor the increase of the PAH relative abundance. However, dust evolution was not found in a previous study made by Paradis et al. (2011a). We investigated the difference in the results between this latter study and the present one and concluded that the absence of the ionized gas phase in the dust decomposition, combined with the inclusion of the dark gas (highly correlated to the atomic phase) in the previous study, significantly affects the shape of the SEDs at small scale. The reason is that at small scale and at $4^{\prime}$ angular resolution the number of pixels is significantly lower than at $1^{\prime}$ angular resolution, which has an impact on the SED and the derived parameters.

For simplicity we name VSG as the dust component responsible of the MIR emission (originating from DBP90). This component corresponds to graphites in DL07 and small amorphous carbons in MC11. In AJ13, the amorphous carbon (a-C) component includes PAHs and does not allow us to analyze each component separately. Our analysis does not show any signs of disappearance of VSG that could result from coagulation on the BGs. However, this process could occur only at very high density, something that we cannot probe in this analysis, or could apply to specific cases and not to the overall molecular clouds. Another explanation could be that molecular clouds in the LMC have different dust properties as compared to those in the Milky Way.

The use of various dust models confirms different properties in the ionized gas: an increase of the dust temperature, an increase of the VSG relative abundance and the relative intensity of the NIR-continuum, and a decrease of the PAH relative abundance. We attribute these differences in the PAH and VSG abundances to different locations of grain formation: VSGs could be formed in HII regions rather than in molecular clouds, whereas PAHs could be formed in the molecular phase of cold and dense clouds. The NIR-continuum does not seem to originate from any single dust component included in the dust models. We also showed that a correlation exists between the relative intensity of the continuum and the intensity of the radiation field. We therefore do not favor the hypothesis of $\mathrm{PAH}$ molecules as carriers of the NIR continuum, nor circumstellar disks as possible candidates. We do not reject the possibility that another small dust component could explain this continuum, such as small carbon molecules heated to high temperatures as proposed by Duley (2009).

Acknowledgements. We are grateful to the referee for his/her careful reading and for all the suggestions which helped to improve the quality of the manuscript. We acknowledge the use of the DustEM package. M.J. acknowledges the support of the Academy of Finland Grant No. 285769.

\section{References}

Aniano, G., Draine, B. T., Calzetti, D., et al. 2012, ApJ, 756, 138 Bernard, J.-P., Reach, W. T., Paradis, D., et al. 2008, AJ, 136, 919 Bot, C., Ysard, N., Paradis, D., et al. 2010, A\&A, 523, A20 Boulanger, F., Abergel, Y. A., Bernard, J.-P., et al. 1996, A\&A, 312, 256 Chastenet, J., Bot, C., Gordon, K. D., et al. 2017, A\&A, 601, A55 Compiègne, M., Verstraete, L., Jones, A., et al. 2011, A\&A, 525, A103 Demyk, K., Mény, C., Lu, X.-H., et al. 2017, A\&A, 600, A123

Désert, F.-X., Boulanger, F., \& Puget, J.-L. 1990, A\&A, 237, 215 Dickinson, C., Davies, R. D., \& Davis, R. J. 2003, MNRAS, 341, 369 Draine, B. T., \& Li, A. 2001, ApJ, 551, 807

Draine, B. T., \& Li, A. 2007, ApJ, 657, 810

Duley, W. W. 2009, ApJ, 705, 446

Dwek, E., Arendt, R. G., Fixsen, D. J., et al. 1997, ApJ, 475, 565 Fazio, G. G., Hora, J. L., Allen, L. E., et al. 2004, ApJS, 154, 10 Feast, M., 1999, in New Views of the Magellanic Clouds, ed. Y.-H. Chu et al. (San Francisco: ASP), IAU Symp. 190

Flagey, N., Boulanger, F., Verstraete, L., et al. 2006, A\&A, 453, 969 
Fukui, Y., Kawamura, A., Minamidani, T., et al. 2008, ApJS, 178, 56 Galametz, M., Madden, S. C., Galliano, F., et al. 2011, A\&A, 532, A56 Galliano, F., Hony, S., Bernard, J.-P., et al. 2011, A\&A, 536, A88 Gaustad, J. E., McCullough, P. R., Rosing, W., \& Van Buren, D. 2001, PASP, 113,1326

Gordon, K. D., Roman-Duval, J., Bot, C., et al. 2014, ApJ, 797, 85

Hughes, A., Wong, T., Ott, J., et al. 2010, MNRAS, 406, 2065

Israël, F. P., Wall, W. F., Raban, D., et al. 2010, A\&A, 519, A67

Jones, A. P., Fanciullo, L., Köhler, M., et al. 2013, A\&A, 558, A62

Keller, S. C., \& Wood, P. R. 2006, ApJ, 642, 834

Kim, S., Staveley-Smith, L., Dopita, M. A., et al. 2003, ApJS, 148, 473

Kohler, M., Guillet, V., \& Jones, A. 2011, A\&A, 528, A96

Kohler, M., Stepnik, B., Jones, A., et al. 2012, A\&A, 548, A61

Lagache, G., Abergel, A., Boulanger, F., et al. 1999, A\&A, 344, 322

Laureijs, R. J., Clark, F. O., \& Prusti, T. 1991, ApJ, 372, 185

Lee, M. Y., Stanimirovic, S., Murray, C. E., et al. 2015, AJ, 809, 56

Leroy, A., Bolatto, A., Gordon, K., et al. 2011, ApJ, 737, 12

Lis, D. C., Serabyn, E., Zylka, R., \& Li, Y., 2001, ApJ, 550, 761

Lu, N. 2004, ApJS, 154, 286

Meixner, M., Gordon, K. D., Indebetouw, R., et al. 2006, ApJ, 132, 2268

Meixner, M., Galliano, F., Hony, S., et al. 2010, A\&A, 518, L71

Mentuch, E., Abraham, R. G., Glazebrook, K., et al. 2009, ApJ, 706, 1020
Mény, C., Gromov, V., Boudet, N., et al. 2007, A\&A, 468, 171 Paradis, D., Reach, W. T., Bernard, J.-P., et al. 2009, AJ, 138, 196 Paradis, D., Reach, W. T., Bernard, J.-P., et al. 2011a, AJ, 141, 43 Paradis, D., Paladini, R., Noriega-Crespo, A., et al. 2011b, ApJ, 735, 6 Paradis, D., Paladini, R., Noriega-Crespo, A., et al. 2012, A\&A, 537, A113 Planck Collaboration XI. 2014, A\&A, 571, A11

Planck Collaboration XXVIII. 2016, A\&A, 594, A28

Planck Collaboration Int. XVII. 2014, A\&A, 566, A55

Poglitsch, A., Waelkens, C., Geis, N., et al. 2010, A\&A, 518, L2

Reach, W. T., Megeath, S. T., Cohen, M., et al. 2005, PASP, 117, 978

Rieke, G. H., Young, E. T., Engelbracht, C. W., et al. 2004, ApJS, 154, 25

Roman-Duval, J., Gordon, K. D., Meixner, M., et al. 2014, ApJ, 797, 86

Sandstrom, K. M., Bolatto, A. D., Draine, B. T., et al. 2010, ApJ, 715, 701

Sandstrom, K. M., Bolatto, A. D., Bot, C., et al. 2012, ApJ, 744, 20

Sellgren, K., Werner, M. W., \& Dinerstein, H. L. 1983, ApJ, 271, 13

Spitzer, L. 1978, Physical Processes in the Interstellar Medium (New York: Wiley-Interscience

Staveley-Smith, L., Kim, S., Calabretta, M. R., et al. 2003, MNRAS, 339, 87

Stepnik, B., Abergel, A., Bernard, J.-P., et al. 2003, A\&A, 398, 551

Tibbs, C., Paladini, R., Cleary, K., et al. 2016, MNRAS, 456, 2290

Wong, T., Hughes, A., Ott, J., et al. 2011, ApJS, 197, 16

Ysard, N., Abergel, A., Ristorcelli, I., et al. 2013, A\&A, 559, A133

Ysard, N., Jones, A. P., Demyk, K., et al. 2018, A\&A, 617, A124 


\section{Appendix A: Results when including the dark gas in the dust decomposition}
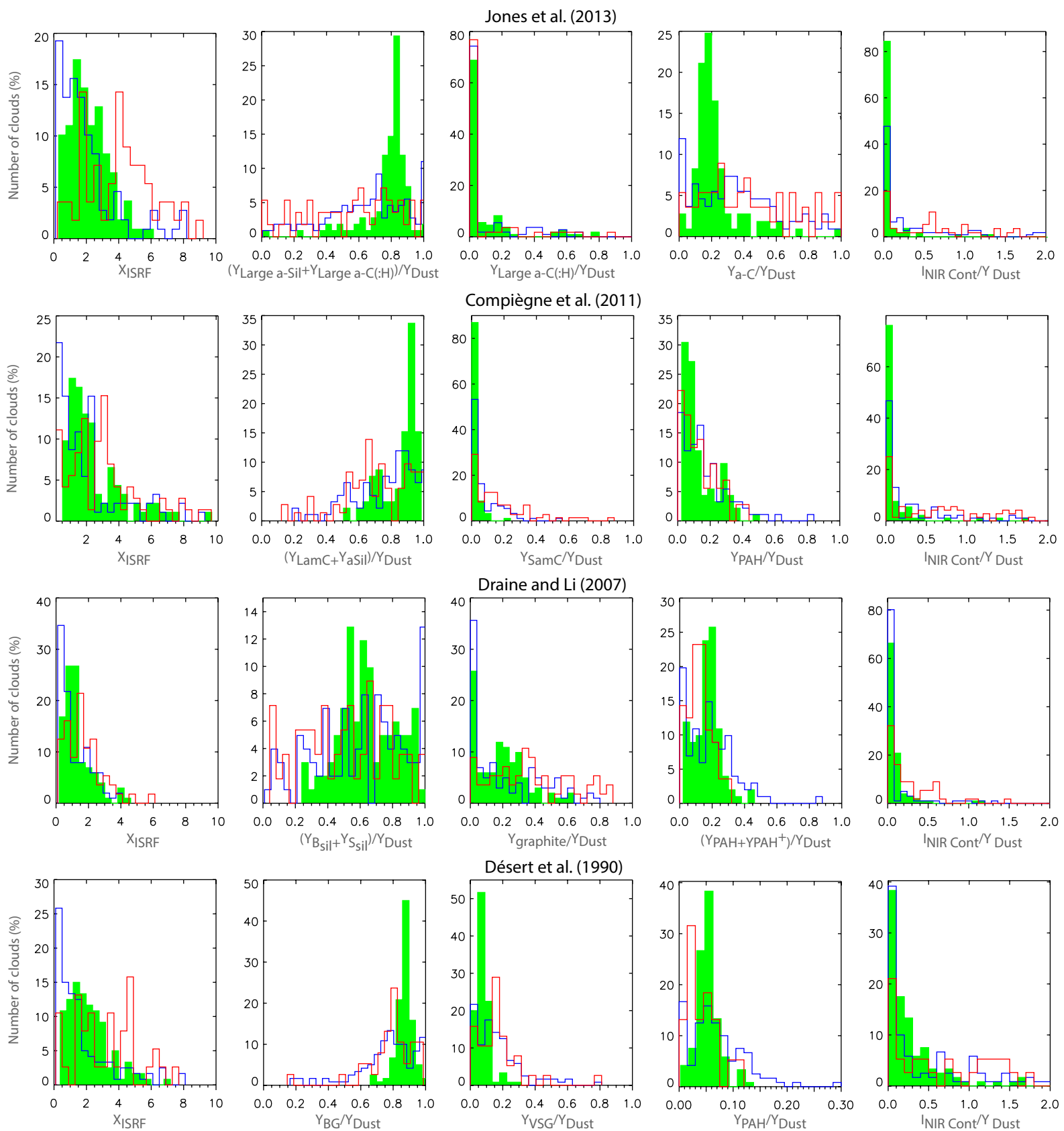

Fig. A.1. Histograms of the parameters derived for each dust model (relative dust abundances, intensity of the radiation field, and relative intensity of the NIR continuum, see Sect. 4.2) in the different gas phases (atomic in green, molecular in blue, and ionized in red), when including the dark gas phase in the decomposition of the dust emission (see Sect. 3). We observe the same trends as in Fig. 10: a decrease of the intensity of the radiation field in the molecular phase and an increase in the ionized phase, as compared to the atomic phase; an increase of the PAH relative abundance in the molecular phase; an increase of the VSG relative abundance in the ionized phase and in the molecular one (that was not observed in Fig. 10); and a more extended distribution of the NIR continuum in the ionized phase as compared to the other phases. 

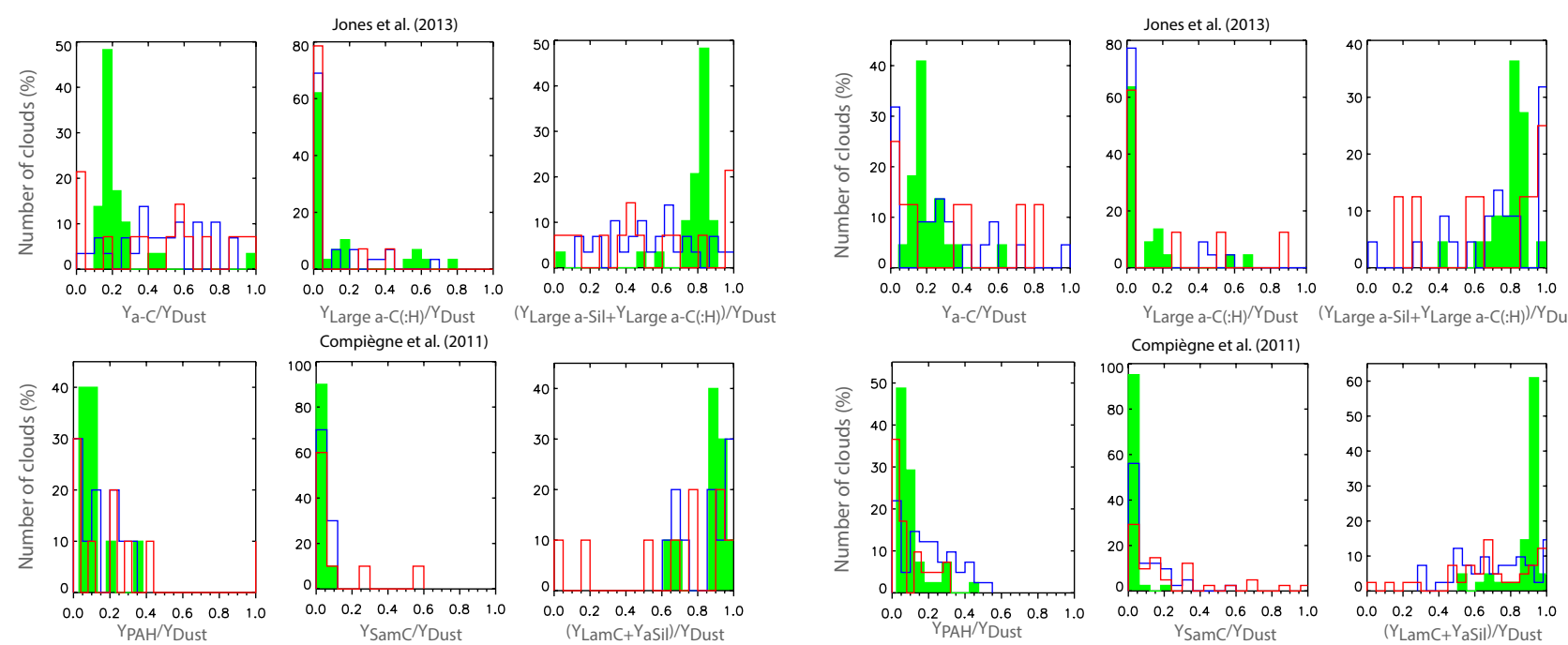

$\begin{array}{lllllll}0.0 & 0.2 & 0.4 & 0.6 & 0.8 & 1.0\end{array}$

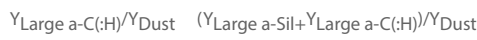
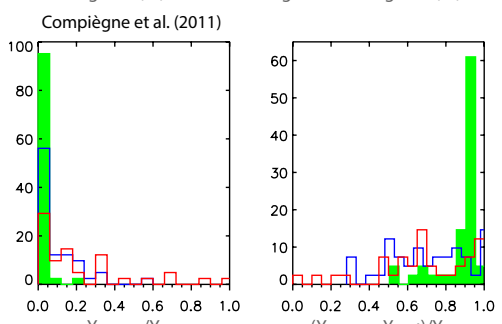

Draine and Li (2007)
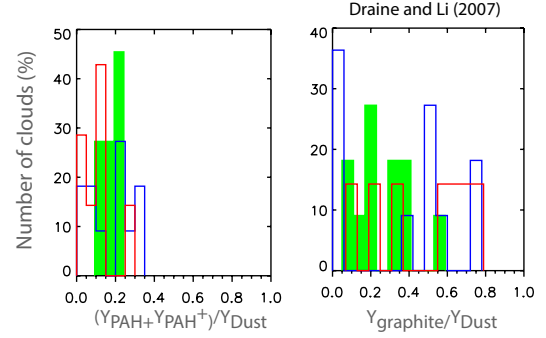

$\begin{array}{ccccc}0.0 & 0.2 & 0.4 & 0.6 & 0.8 \\ & Y \text { graphite } / Y_{\text {Dust }}\end{array}$
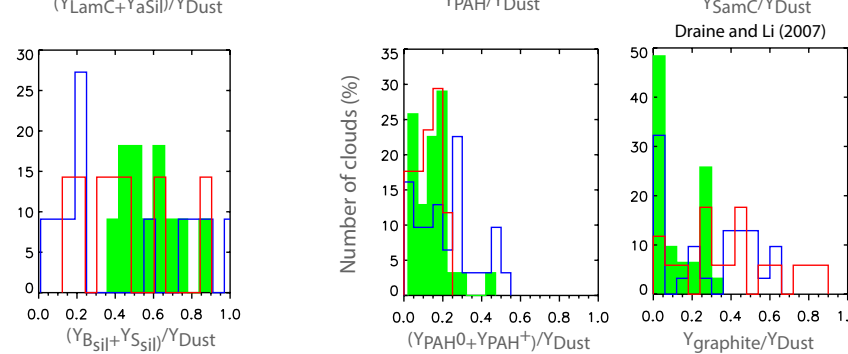

(Y
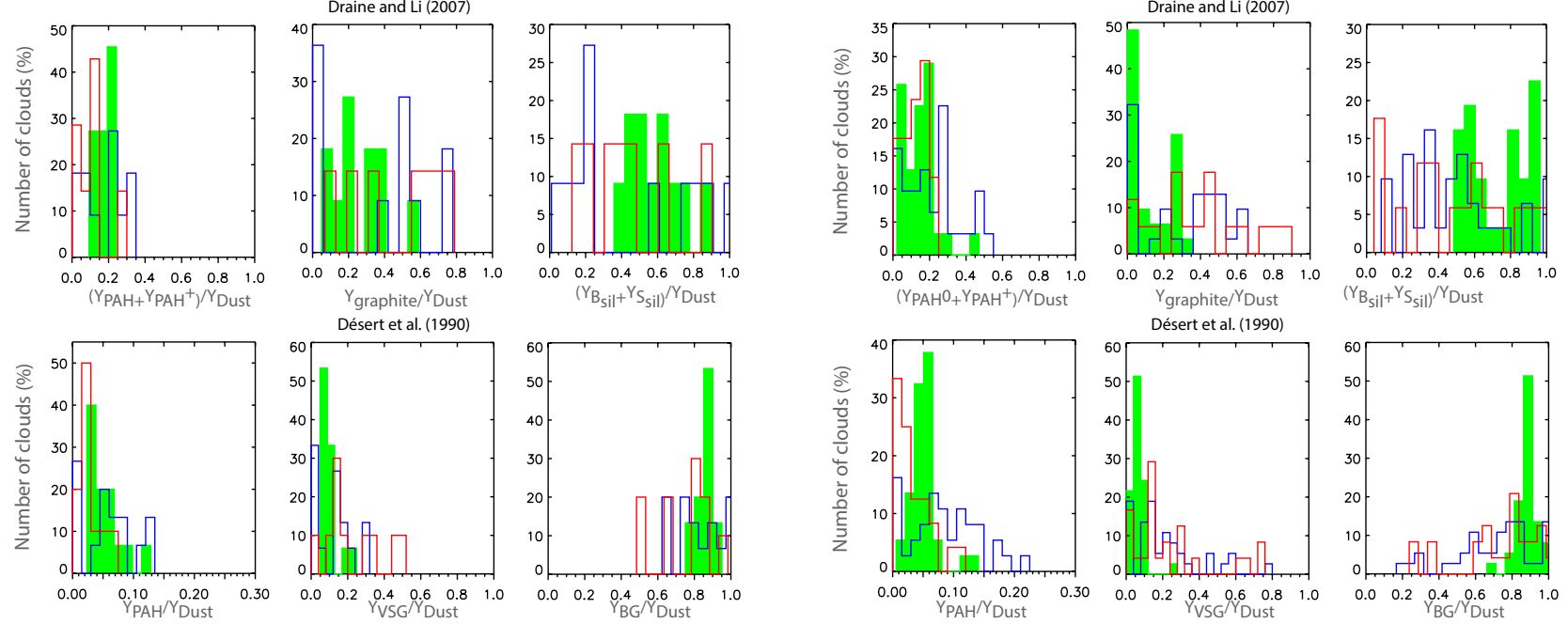

Fig. A.2. Histograms of the relative abundances of the dust components for clouds with similar dust temperatures in the atomic and molecular phases (case 1 as defined in Sect. 7.1) on the left, and for clouds with significantly colder dust temperatures in the molecular than in the atomic phases (case 2) on the right. The atomic, molecular, and ionized phases are shown in green, blue, and red, respectively. The dark gas phase has been taken into account in the decomposition of the dust emission (see Sect. 3). As evidenced in Fig. 11, we notice an enhancement of the PAH relative abundance in the molecular phase (as compared to the atomic phase) for clouds in case 2 as compared to clouds in case 1. The VSG relative abundance is also increased in the ionized phase in both cases, as well as in the molecular phase (that was not observed in Fig. 11). 